\title{
Colored SUSY and R-hadron physics in the ATLAS detector
}

QCHS-06

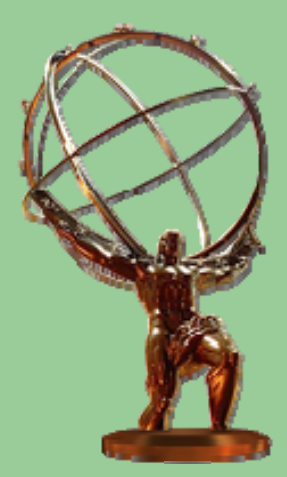

Rasmus Mackeprang, rasmack@nbi.dk 


\section{Outline}

- SUSY reminders using SPS1a as an example

- Light $\tilde{t}$ scenario

- R-hadron phenomenology

- R-hadron measurements 


\section{Basic SUSY phenomenology}

- Using SPS1a

- Defined in mSUGRA using parameters:

$$
\begin{aligned}
& m_{0}=-A_{0}=0.4 m_{\frac{1}{2}}=100 \mathrm{GeV} \\
& \tan \beta=10, \mu>0
\end{aligned}
$$

- SUSY spectrum has $m_{\tilde{q}}>m_{\tilde{\chi}_{2}^{0}}>m_{\tilde{l}}>m_{\tilde{\chi}_{1}^{0}}$ so we have the squark decaying through a neutralino and a slepton to the $\chi_{1}^{0}$ LSP which is stable and escapes the detector. 


\section{Cascade decays}

O

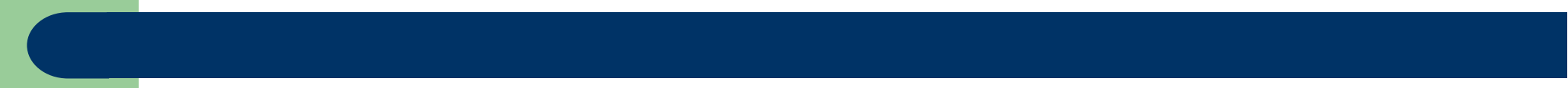

- Sequential decay:

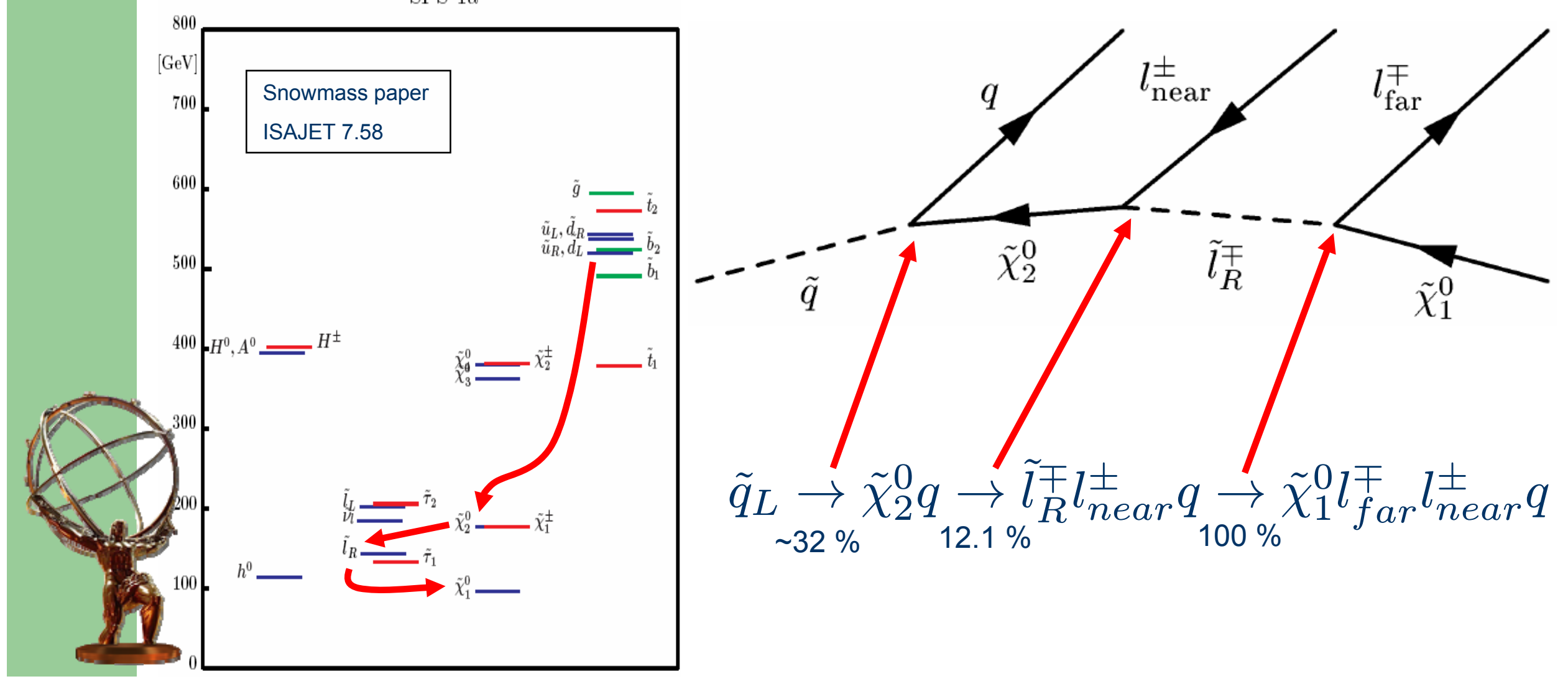


Slide 4

RM1 Figures from JHEP09(2000)004

Rasmus Mackeprang, 8/2/2006 


\section{Kinematic variables}

- As the $\chi_{1}^{0}$ escapes the detector one may form these invariants: $m_{l l}, m_{l_{1} q}, m_{l_{2} q}, m_{l l q}$

- Note that we don't know "near" from "far" lepton $\rightarrow$ Maximize/minimize in combination choice.

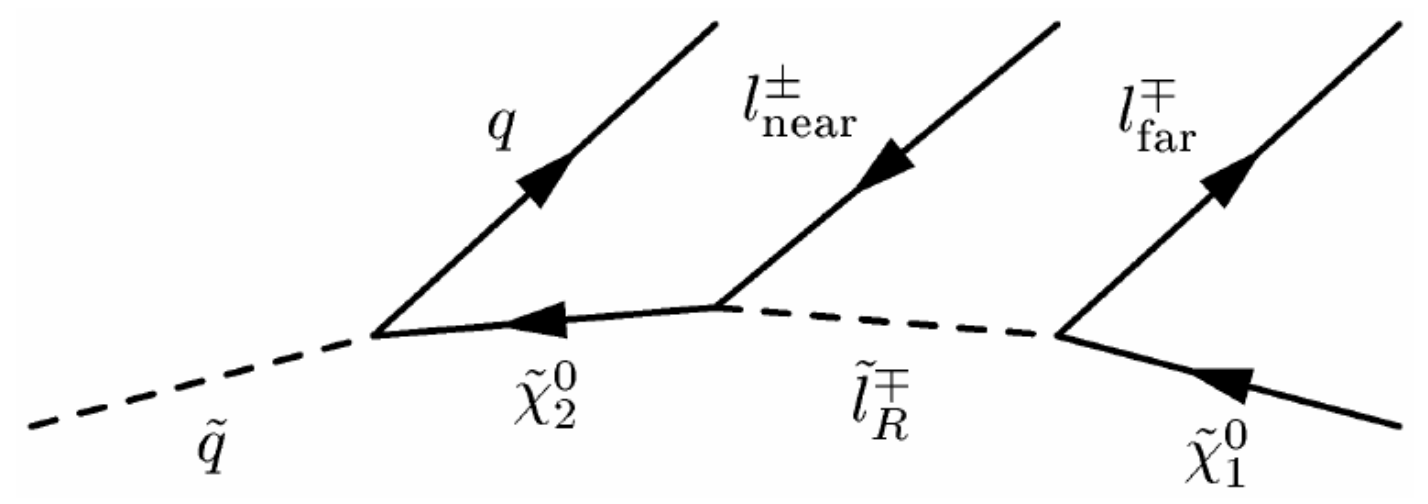




\section{Kinematic edges and masses}

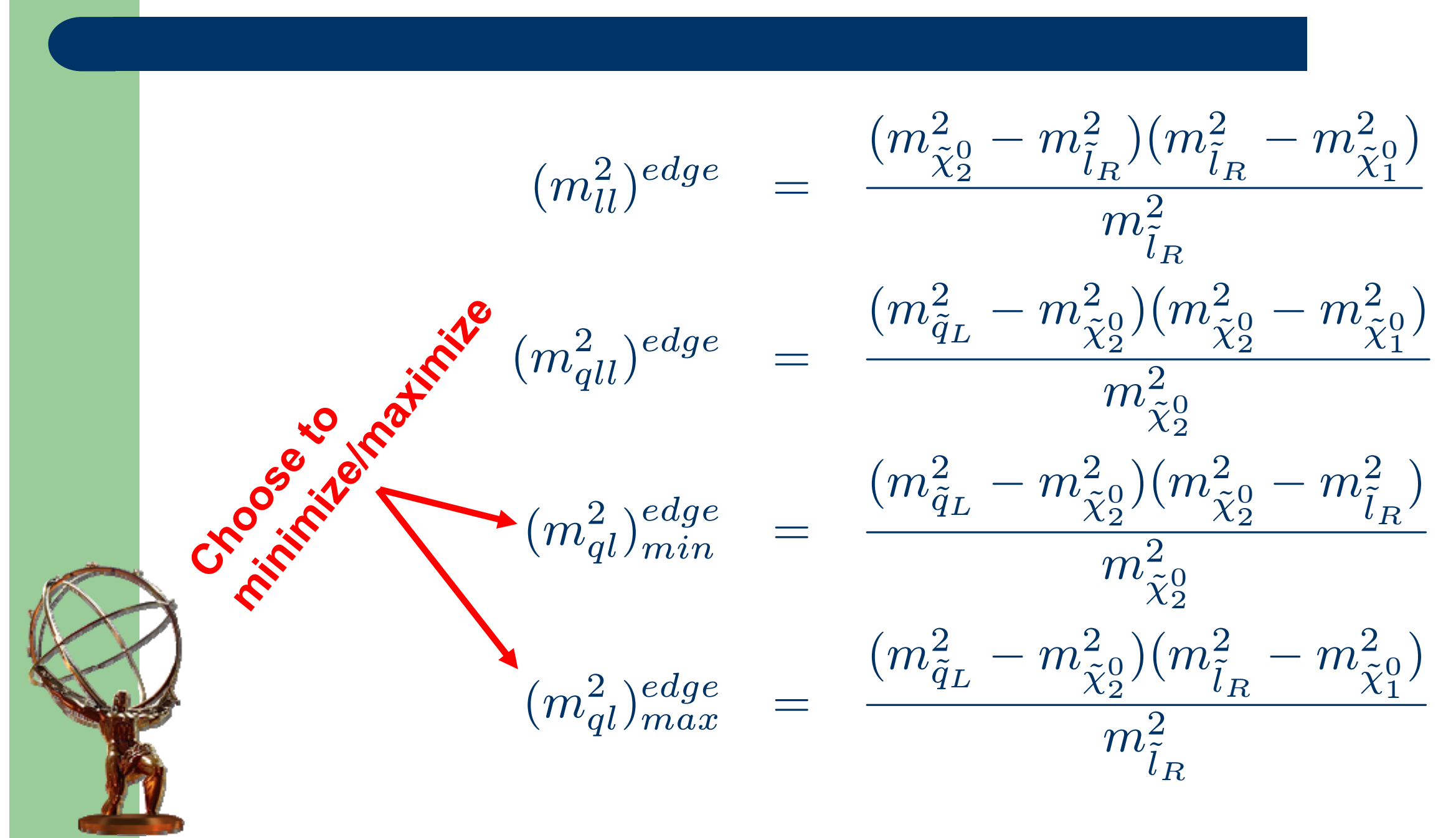




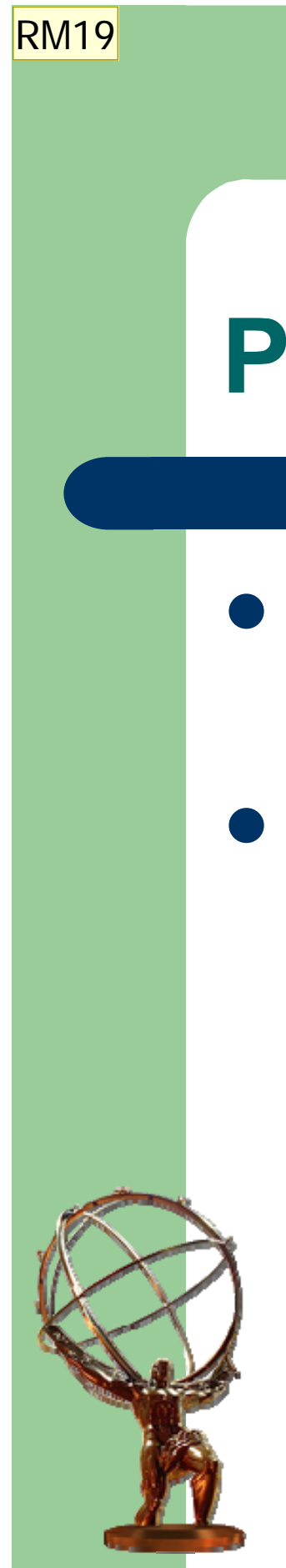

Production and analysis cuts

- Events produced w PYTHIA $6.2\left(100 \mathrm{fb}^{-1}\right)$ and run through a fast detector simulation

- Analysis cuts:

$\star$ At least four jets satisfying

$$
p_{t, 1}>150 \mathrm{GeV}, p_{t, 2}>100 \mathrm{GeV}, p_{t, 3}>50 \mathrm{GeV}
$$

$\star M_{\text {eff }} \equiv E_{t, \text { miss }}+p_{t, 1}+p_{t, 2}+p_{t, 3}+p_{t, 4}>600 \mathrm{GeV}$

$\star E_{t, m i s s}>\max \left(100 \mathrm{GeV}, 0.2 M_{e f f}\right)$

$\star$ Two isolated leptons (not $\tau$ ) of opposite charge

but same flavour (OSSF) with

$p_{t}(l)>20 \mathrm{GeV}$ and $p_{t}(l)>10 \mathrm{GeV}$ 


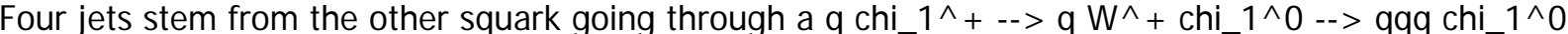
Rasmus Mackeprang, 8/29/2006 


\section{Lepton-lepton invariant mass}

- Before and after OS-OF lepton background subtraction:
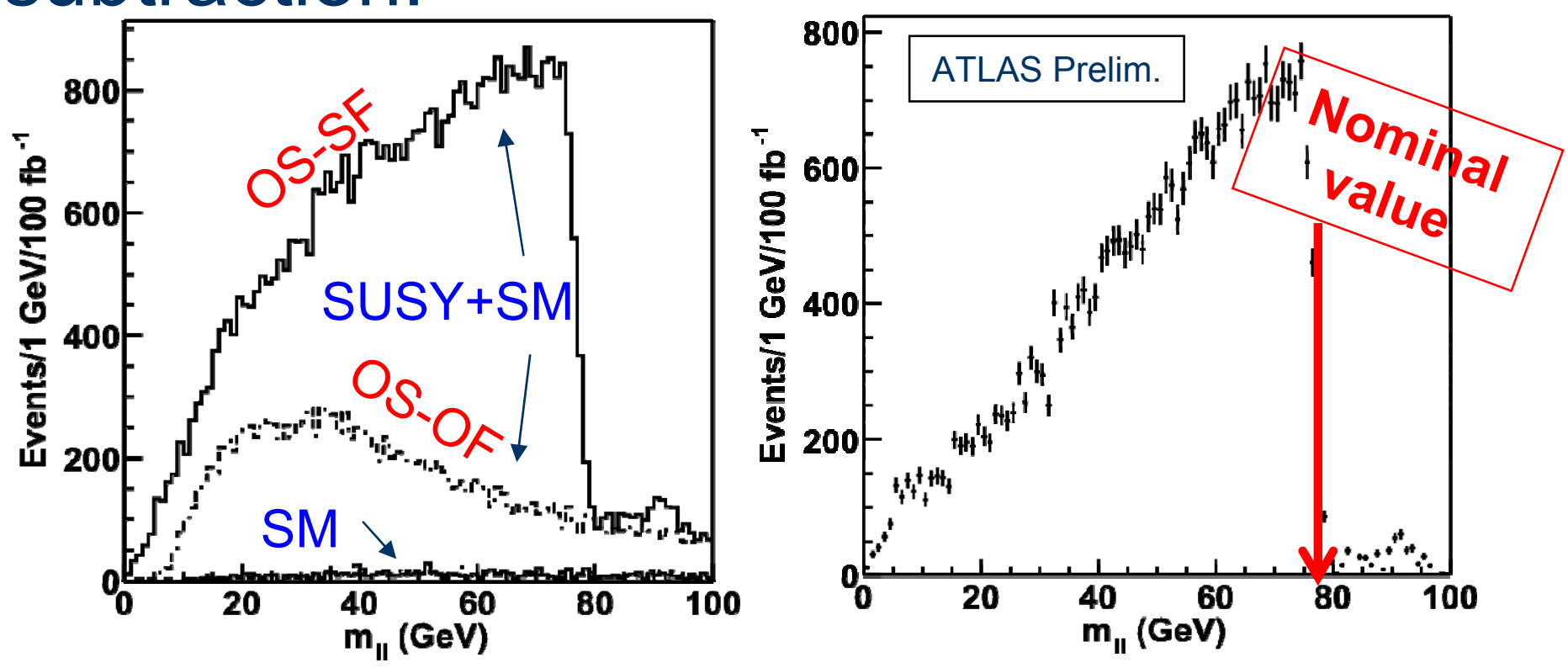

$m(l l)^{\text {edge }}: 77.077^{\text {nominal }}$

vs. $77.024^{\mathrm{rec}} \pm 0.08^{\mathrm{syst}} \pm 0.05^{\mathrm{stat}}$ 
RM17 Following plots include subtraction

Rasmus Mackeprang, 8/28/2006 

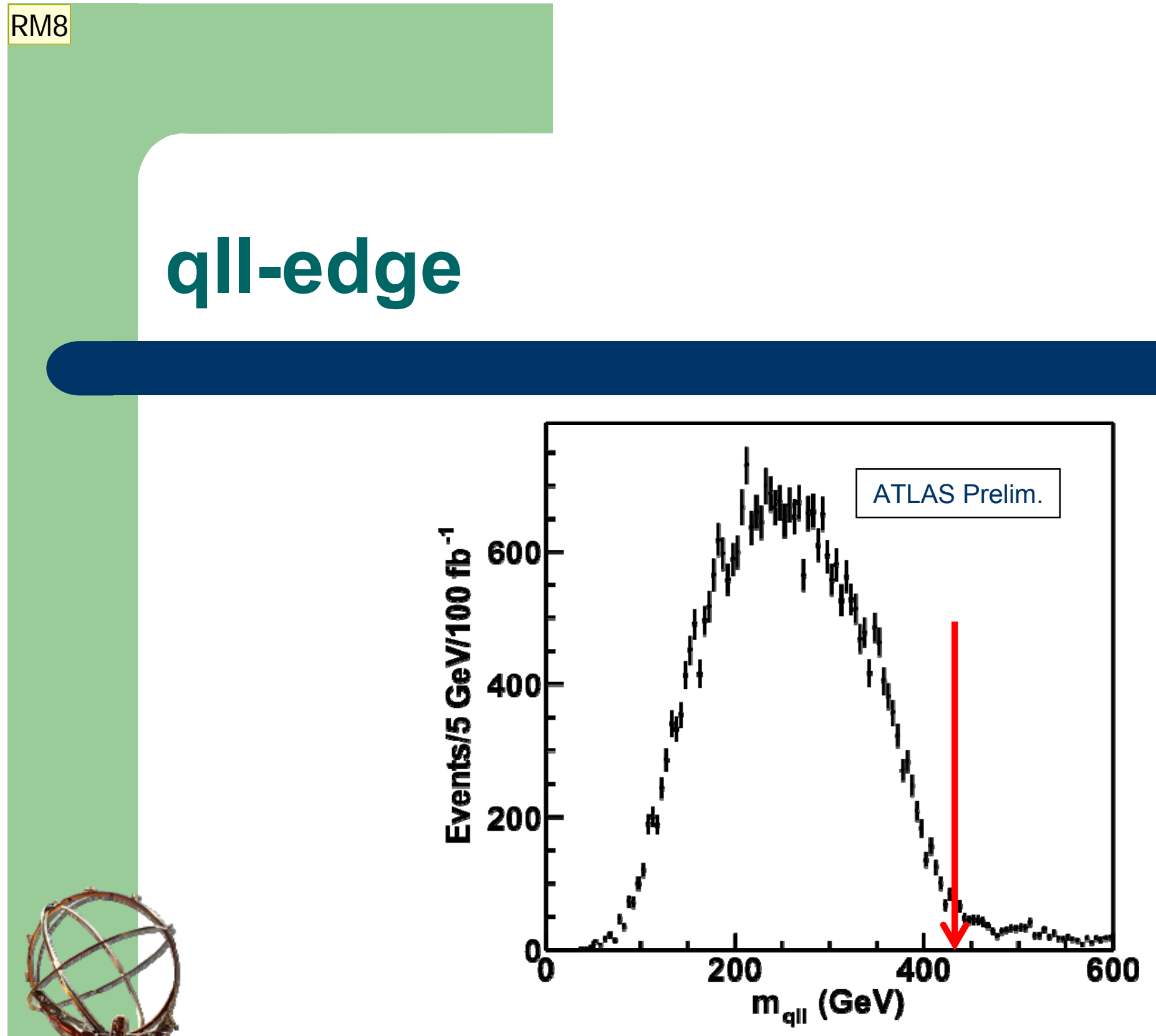

$m(q l l)^{\text {edge }}: 431.1^{\text {nominal }}$ vs. $431.3^{\text {rec }} \pm 4.3^{\text {syst }} \pm 2.4^{\text {stat }}$ 
J ust a visual confirmation that the method works

Rasmus Mackeprang, 8/4/2006 


\section{ql}
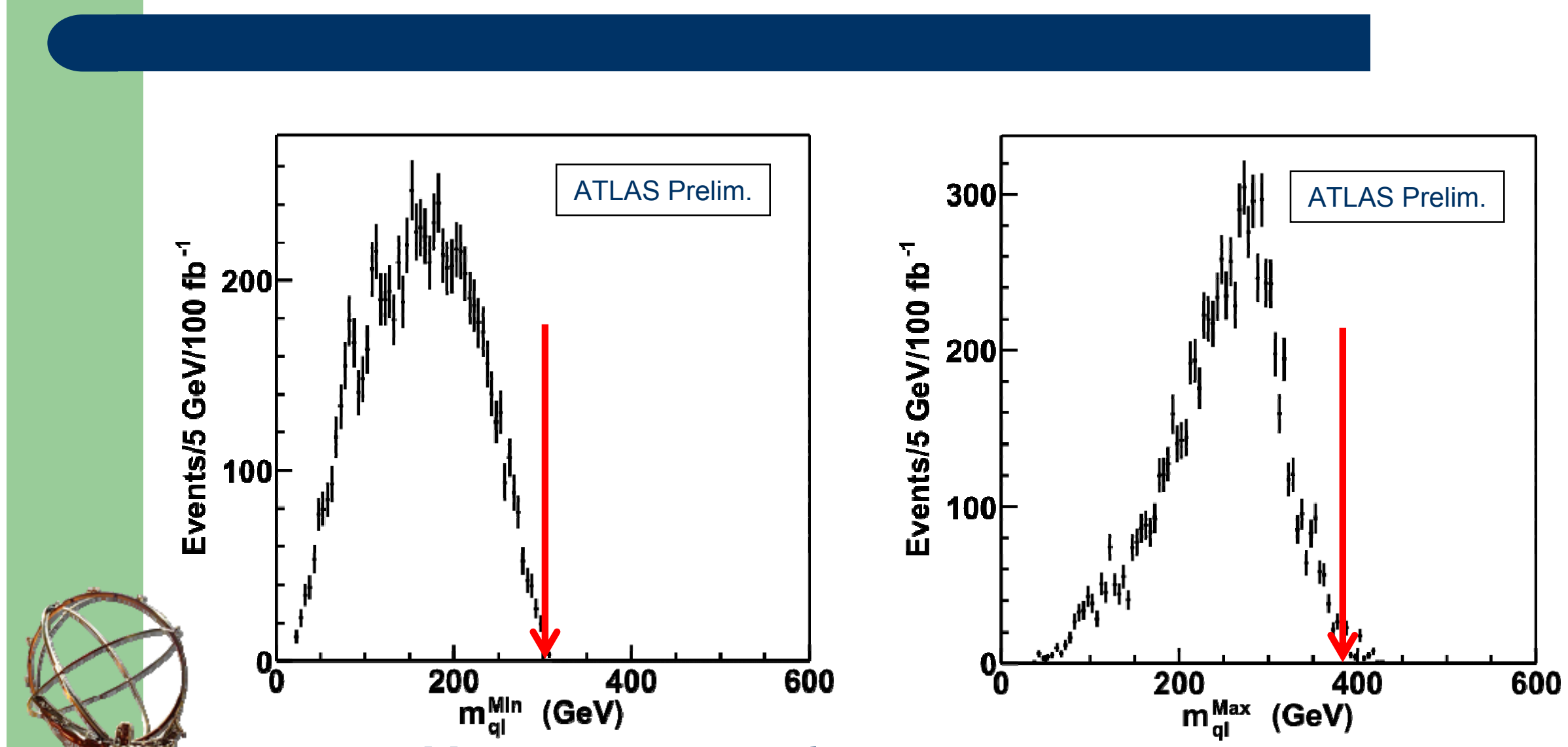

$$
\begin{aligned}
& m(q l)^{\mathrm{Max}}: 380.3^{\text {nominal }} \text { vs. } 379.4^{\text {rec }} \pm 3.8^{\text {syst }} \pm 1.8^{\text {stat }} \\
& m(q l)^{\text {Min }}: 302.1^{\text {nominal }} \text { vs. } 300.8^{\text {rec }} \pm 3.0^{\text {syst }} \pm 1.5^{\text {stat }}
\end{aligned}
$$




\section{Mass measurements}

- Measure a number of parameters and constraints:

$$
\begin{aligned}
& m_{l l}^{\max }, m_{l l q}^{\max }, m_{l q}^{l o w}, m_{l q}^{\text {high }}, m_{l l q}^{\min }, m_{l l b}^{\min }, m\left(l_{L}\right)-m\left(\tilde{\chi}_{1}^{0}\right), \\
& \mathrm{m}_{l l}^{\max }\left(\tilde{\chi}_{4}^{0}\right), m_{\tau \tau}^{\max }, m(\tilde{g})-0.99 \times m\left(\tilde{\chi}_{1}^{0}\right), \ldots
\end{aligned}
$$

- Minimize chisquare function:

$$
\chi^{2}=\sum_{j}\left[\frac{E_{j}^{\text {theory }}(\vec{m})-E_{j}^{\text {exp }}}{\sigma_{j}^{\text {exp }}}\right]^{2}
$$




\section{Atlas Performance Estimate}

- Perform $10^{5}$ "Atlas experiments" and study the RMS of the resulting distributions of parameters:

\begin{tabular}{clll}
\hline$\Delta m_{\tilde{\chi}_{1}^{0}}$ & 4.8 & $\Delta m_{\tilde{l}_{R}}$ & 4.8 \\
$\Delta m_{\tilde{\chi}_{2}^{0}}$ & 4.7 & $\Delta m_{\tilde{q}_{L}}$ & 8.7 \\
$\Delta m_{\tilde{b}_{1}}$ & 7.5 & $\Delta m_{\tilde{g}}$ & 8.0 \\
$\Delta m_{\tilde{q}_{R}}$ & 11.8 & $\Delta m_{\tilde{b}_{2}}$ & 7.9 \\
$\Delta m_{\tilde{l}_{L}}$ & 5.0 & $\Delta m_{\tilde{\chi}_{4}^{0}}$ & 5.1 \\
\hline rrors in GeV. & & $\mathrm{L}=300 \mathrm{fb}^{-1}$
\end{tabular}




\section{Light $\tilde{t}$ Analysis}

- Cosmological inspiration

- Discard the demand for unification (no assumptions on SUSY breaking)

- Require matter-antimatter asymmetry to be generated at EW scale (CPV + light $\tilde{t})$

- Fix MSSM parameters using cosmological observables. 


\section{Benchmark Point}

- LHS-2 (Les Houches 2005)

MSSM Parameters $(\mathrm{GeV})$ :

\begin{tabular}{ll} 
1st. 2nd. gen. scalars & 10000 \\
3rd. gen. sleptons & 1000 \\
$m\left(Q_{3}\right)$ & 1500 \\
$m\left(\tilde{t}_{R}\right)$ & 0 \\
$m\left(\tilde{b}_{R}\right)$ & 1000 \\
$A_{b, e, \mu, \tau}$ & 0 \\
$A_{t}$ & $-643 \exp \frac{i \pi}{2}$ \\
$M_{1}$ & 60 \\
$M_{2}$ & 120 \\
$\mu$ & $400 \exp \frac{i \pi}{2}$ \\
$\tan \beta$ & 7 \\
$M(A)$ & 1000 \\
\hline
\end{tabular}

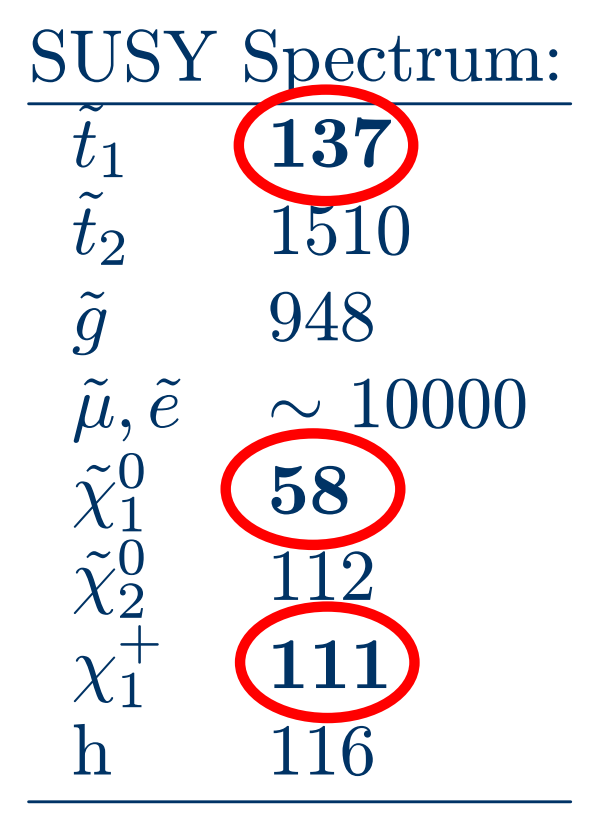

I 


\section{Basic Phenomenology}

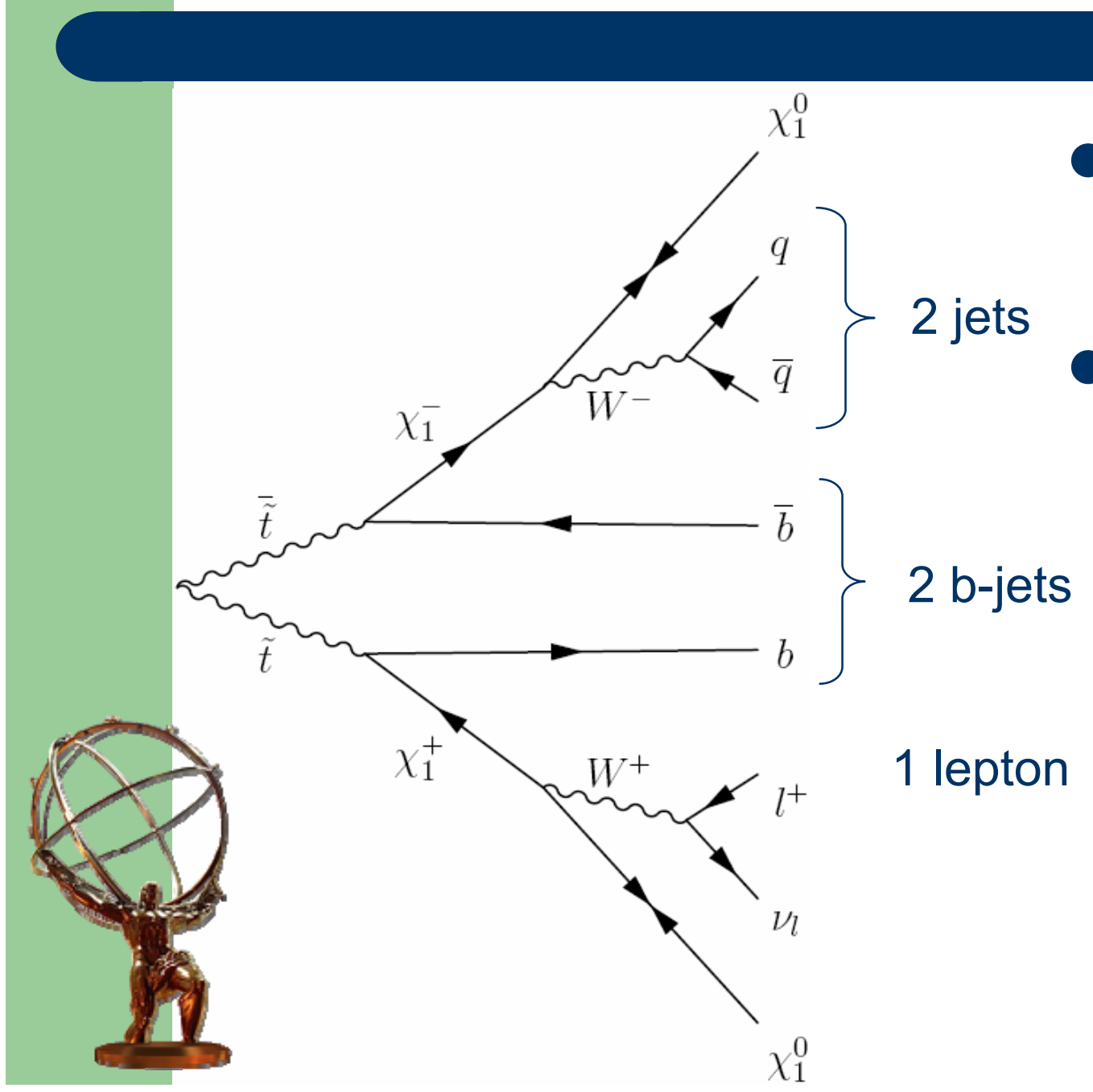

- Missing transverse energy

- Kinematics doesn't match $\mathrm{t} / \mathrm{W}$ production

- Jets too soft

- Invariant mass combinations don't add up. (W's are virtual) 


\section{Basic Cuts}

- One isolated e/ $\mu$ with $p_{t}>20 \mathrm{GeV}$

- $\mathrm{E}_{\mathrm{t} \text {,miss }}>20 \mathrm{GeV}$

- 4 jets with $p_{t}>25 \mathrm{GeV}\left(2\right.$ with $\left.p_{t}>35 \mathrm{GeV}\right)$

- 2 b-jets

Efficiencies:

Signal: $0.47 \% \quad$ tt: $3.3 \% \quad$ Wbbj: $3.1 \%$ b/s ratio $\sim 15:$ (but soft QCD is gone)

Fast simulation study on $1.8 \mathrm{fb}^{-1}$ 


\section{Excluding Ws}
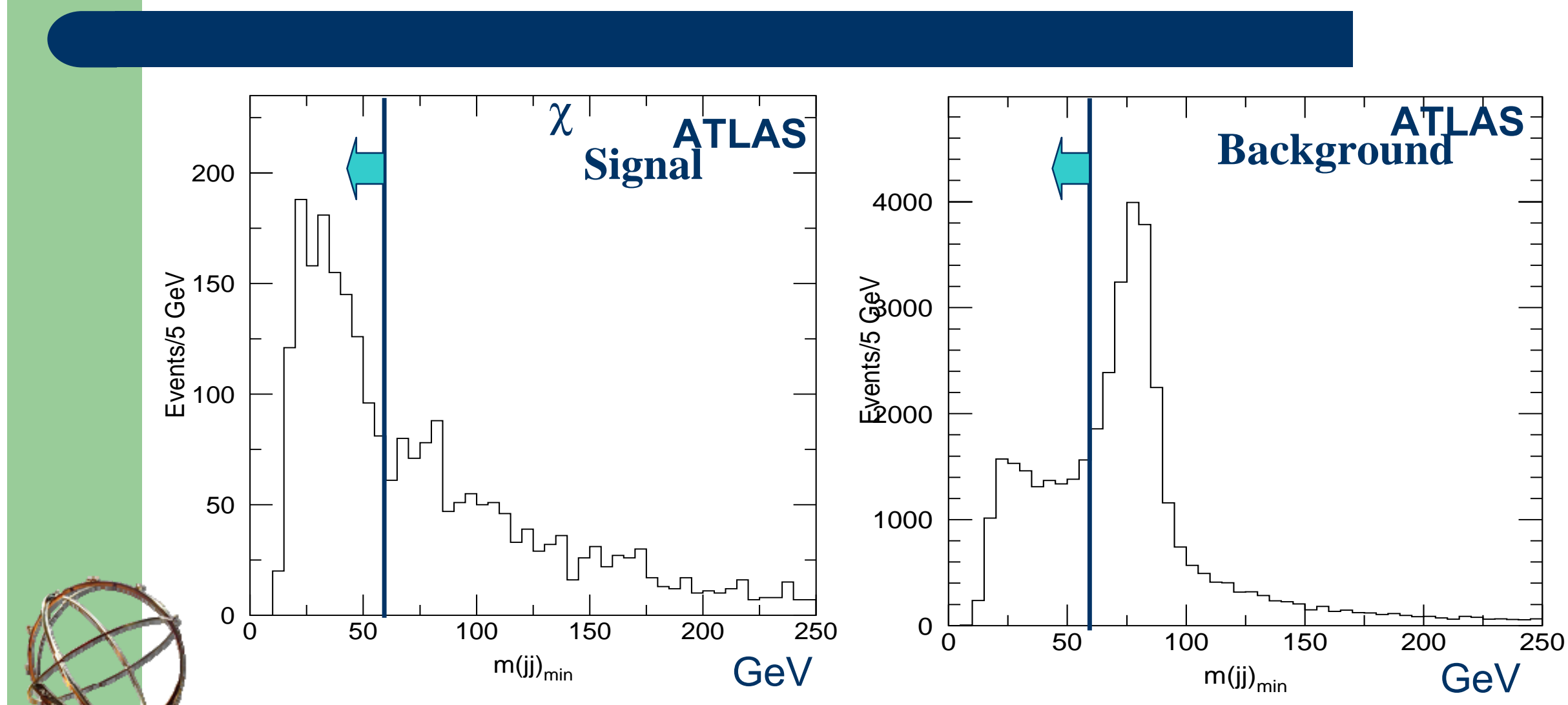

Demanding $\mathrm{m}(\mathrm{jj})_{\min }<60 \mathrm{GeV}$ removes a lot of W background $\rightarrow$ b/s $\sim 10$ 


\section{After background subtraction}
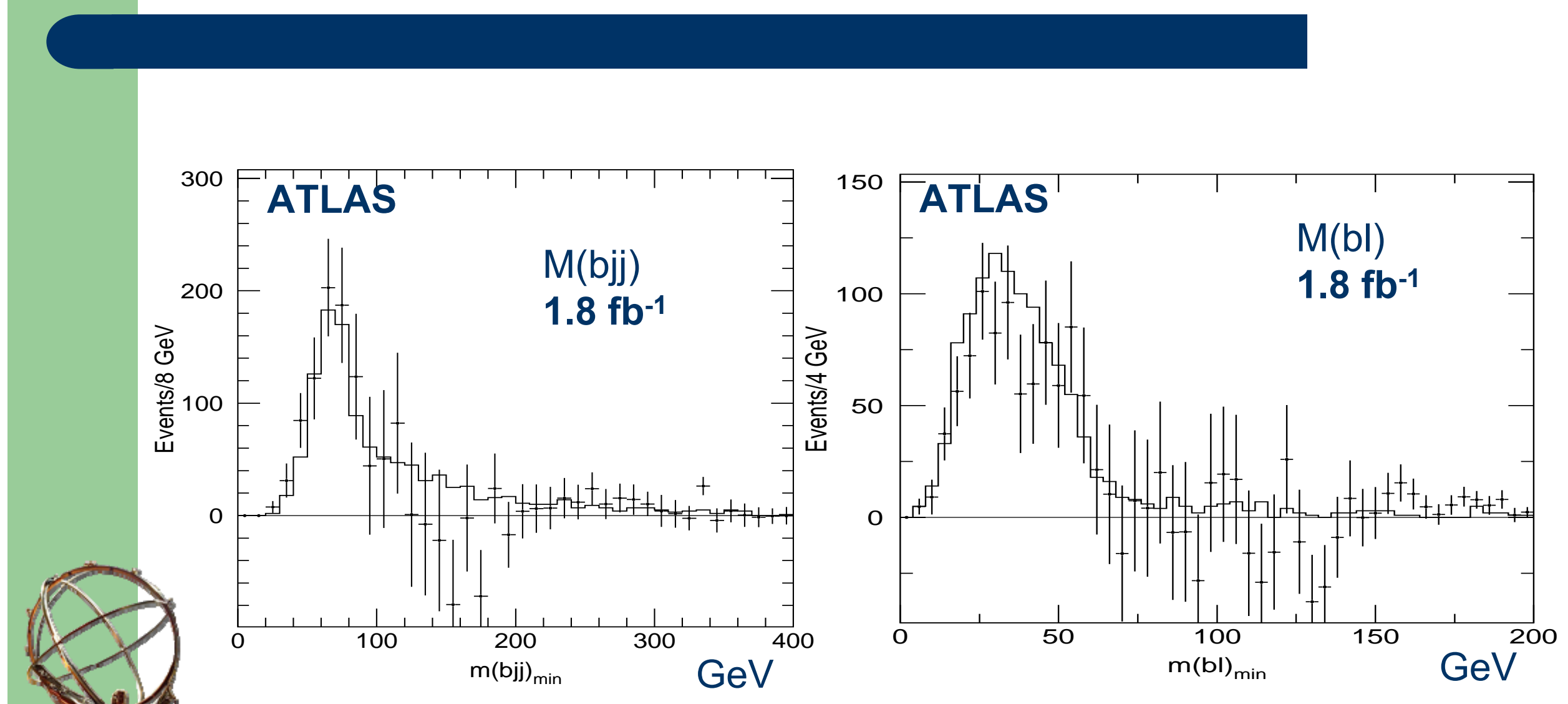

Next step would be fitting masses but that is a Ii somewhat longer story... 


\section{R-hadrons}

- Scenarios with metastable squarks/gluinos

- Coloured SUSY particle binds with quarks to form R-(parity-stable) hadrons. Hence the name...

- Possible scenario is split-SUSY:

- Abandon hierarchy problem

- SUSY broken far above the TeV scale $\rightarrow$ heavy scalars, light Higgs and light fermions

- Long lived gluino 


\section{Interactions of \\ Physics model for R-hadrons}

- Quark system interacts

- Gluino is "just" a reservoir of kinetic energy

- Interactions are thought to be pomeron / reggeon mediated:
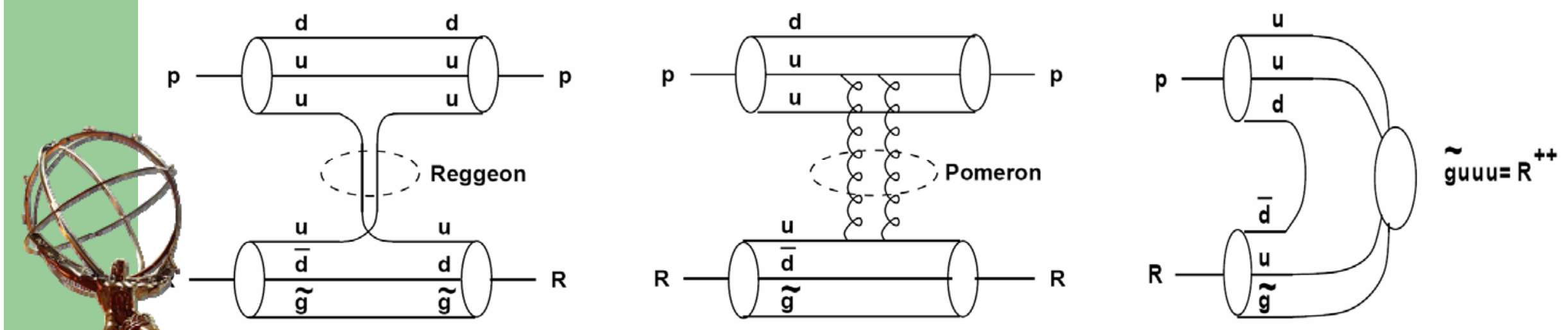

Figure: A.C. Kraan 


\section{Basic Phenomenology}

- R-hadrons can change charge in hadronic interactions.

- R-mesons tend to acquire baryon number

- Cross section ensures hadronic interactions in calorimeter.

- A pure sample of R-hadrons can be selected basically by requiring opposite sign tracks in ID and muon system.

Simulation implemented in Geant3 and Geant4 


\section{Energy loss per collision}

\section{$300 \mathrm{GeV} / \mathrm{c}^{2}$ gluino in iron:}

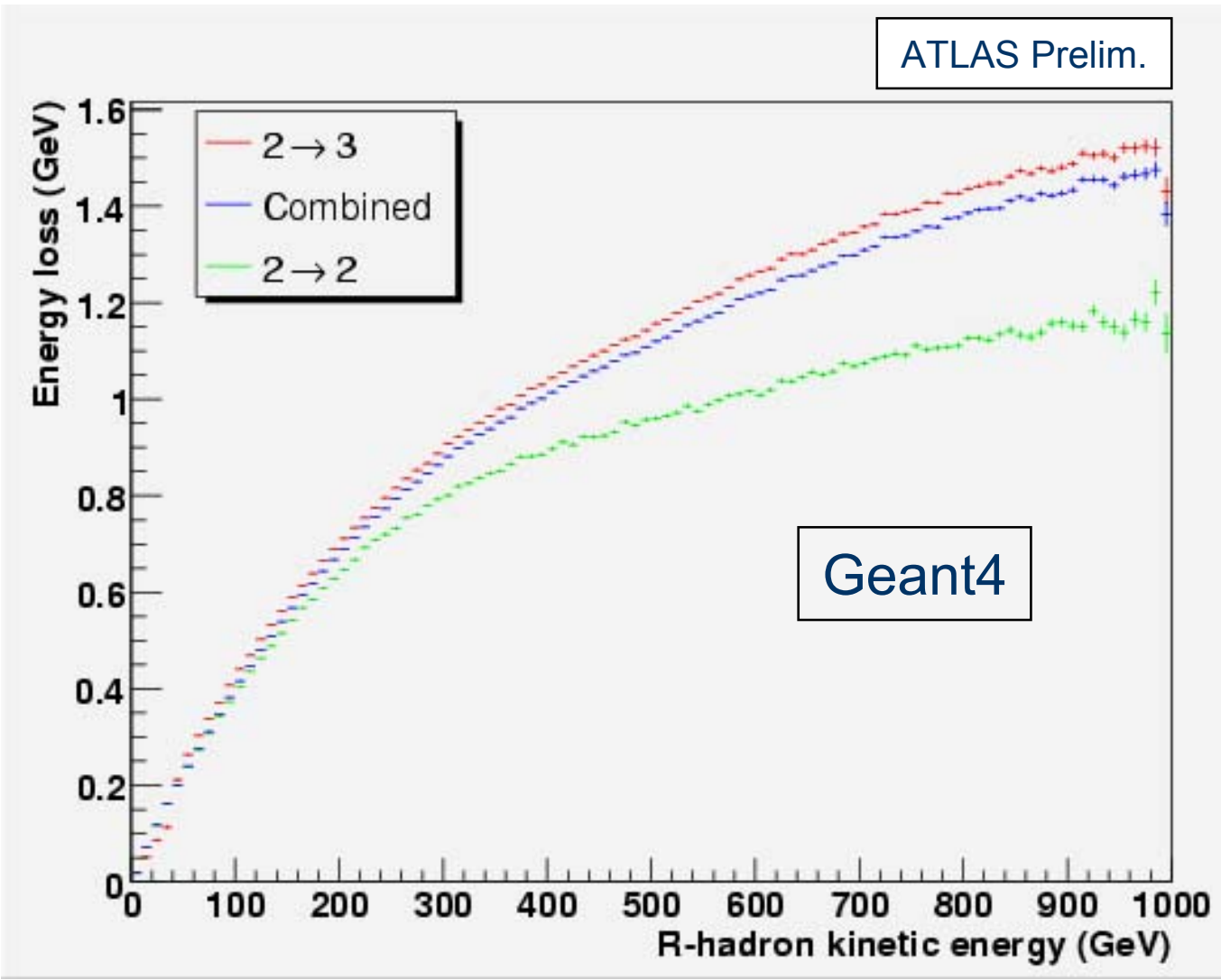




\section{Triggering}

.

- Charged R-hadrons in muon system will pass the single- $\mu$ $6 \mathrm{GeV}$ trigger at even very moderate kinetic energies.

- $\beta>0.7$ satisfies timing.

Losses:

$25 \% @ 300 \mathrm{GeV} / \mathrm{c}^{2}$

$60 \% @ 1 \mathrm{TeV} / \mathrm{c}^{2}$

Additional $\mathrm{O}(50 \%)$ loss from hadronic interactions in muon system
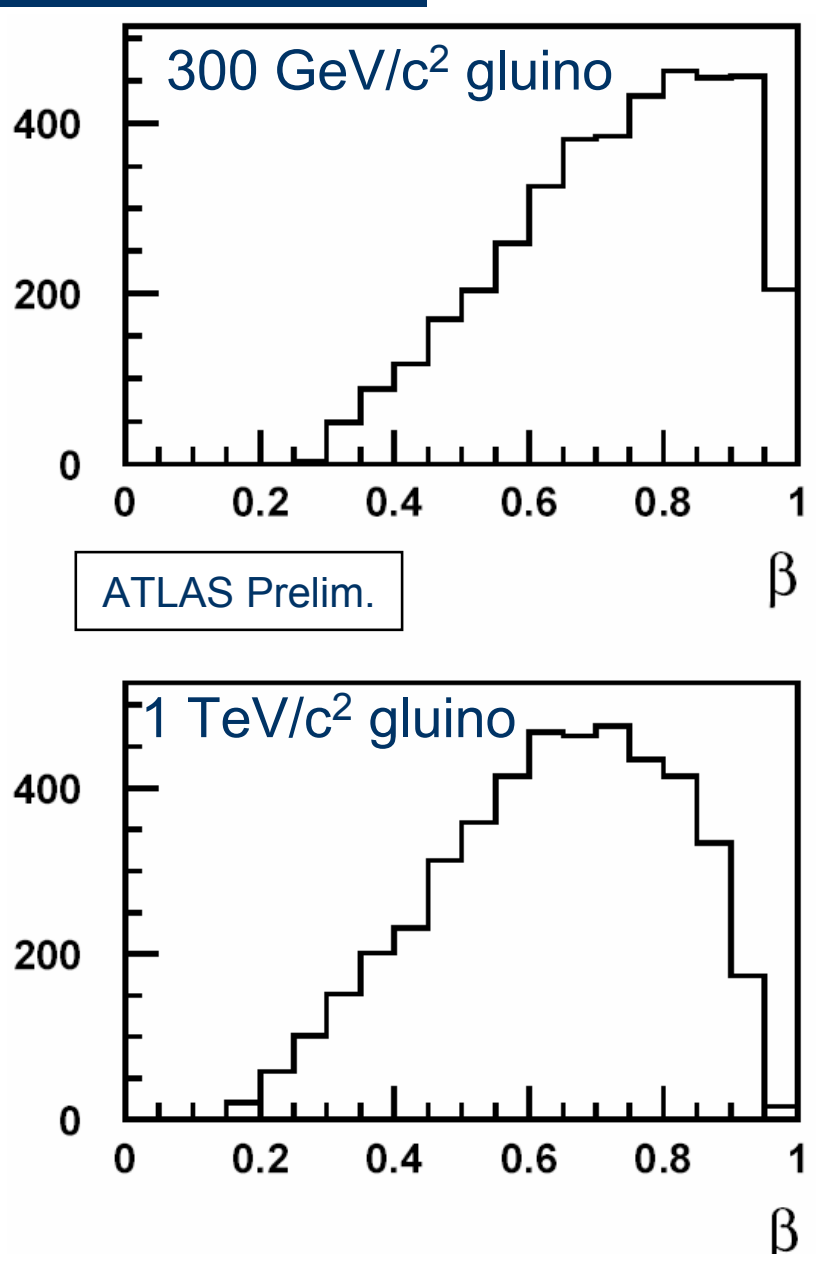


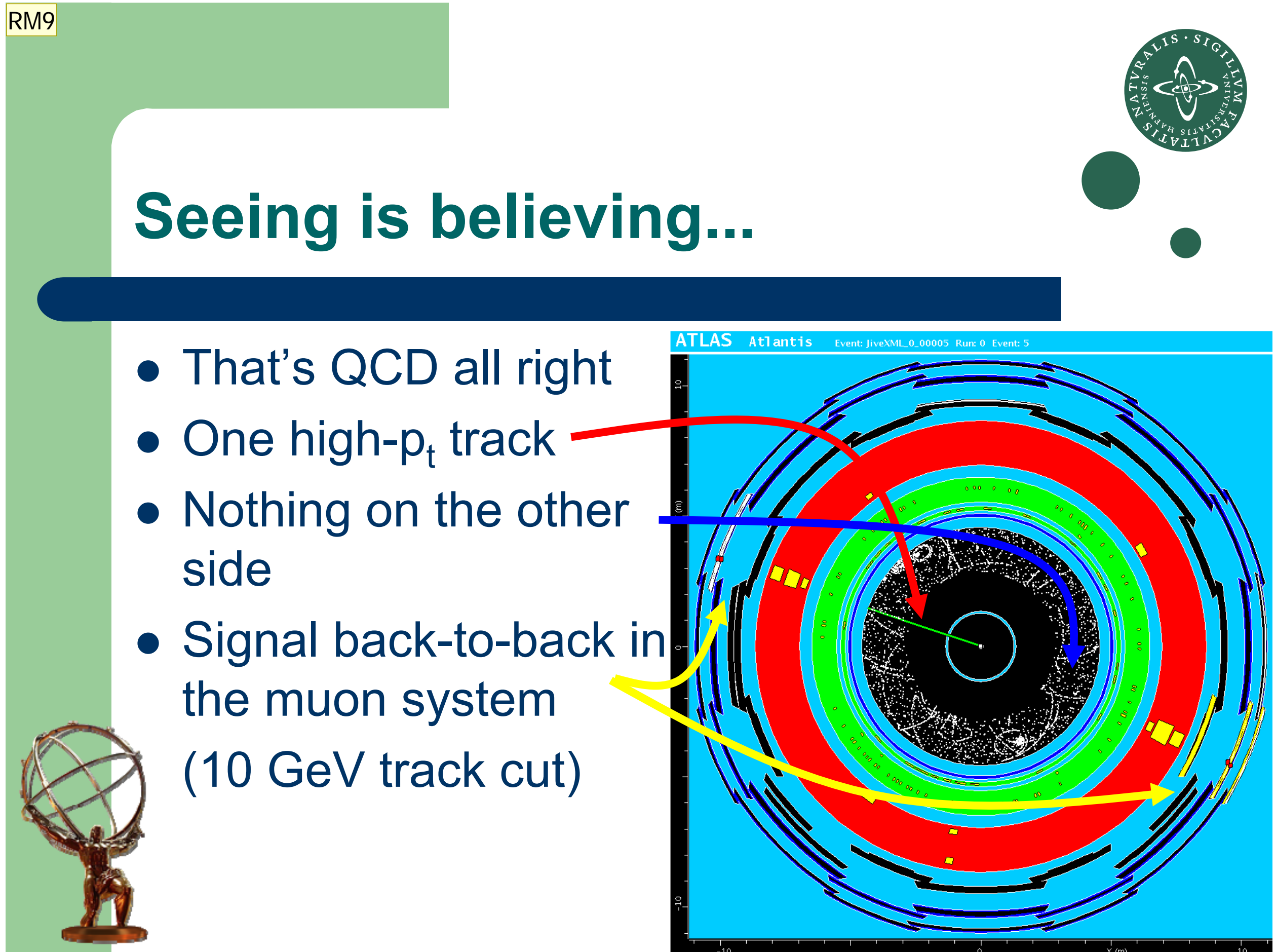


This is just an event display I made for illustrative purposes. I hope it is OK to use it. Rasmus Mackeprang, 8/4/2006 


\section{Charge Flipping Quantified}

- Split SUSY scenario: $\left(2 \mathrm{fb}^{-1}\right)$
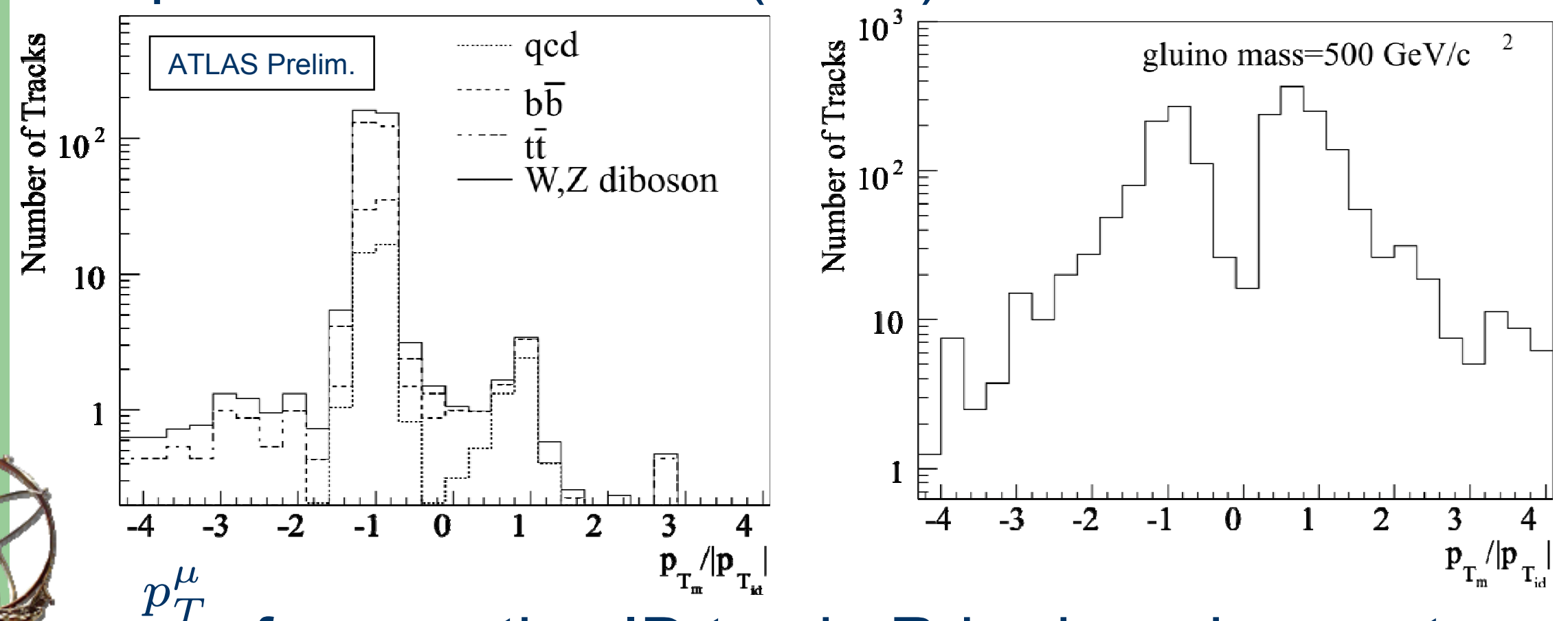
$\frac{p_{T}^{\mu}}{\left|p_{T}^{I D}\right|}$ for negative ID track. R-hadron does not "remember" initial charge. 


\section{R-hadron Mass Measurement}

- Gluino does not decay $\rightarrow$ Mass measurement requires $\beta$-measurement.

- Use drift time in muon chambers:

$T_{i}=T_{0}-\frac{L_{i}}{c \beta}$

$T_{i}$ : Drift time

$T_{0}$ : Time from creation to detection. 


\section{$\beta$-measurement}

- Use $\beta$ as an assumption in track fit.

- Divide events into momentum intervals

ATLAS Prelim.

- Minimize chi-square as:

- Use that

$$
p=m c \beta \gamma
$$

to fit the mass.

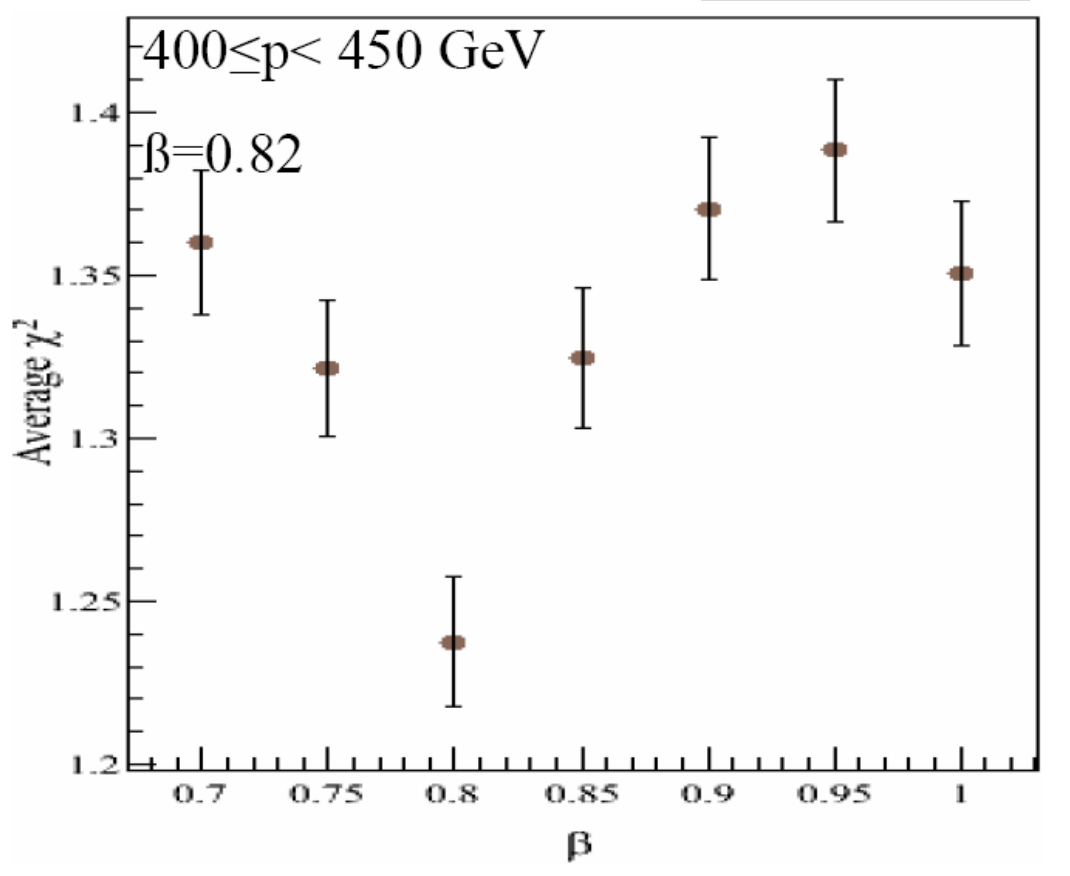




\section{Fitted mass}

- Input gluino mass was $300 \mathrm{GeV} / \mathrm{c}^{2}$

$\Rightarrow$ Mass scale can be determined to

$\mathrm{O}(5-10 \%)$ using simple and generic methods.

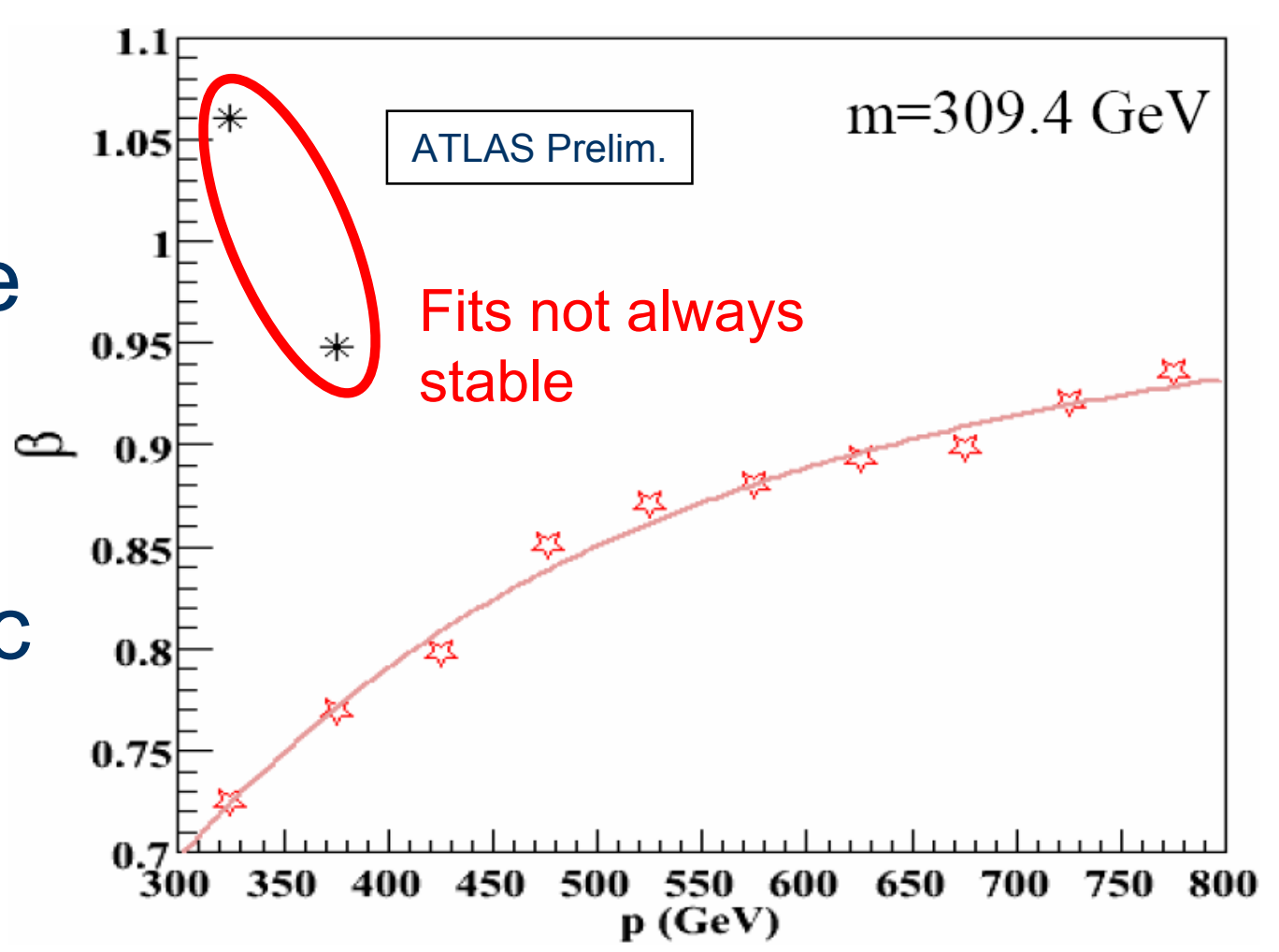




\section{Summary}

- The Atlas detector is sensitive to a broad spectrum of SUSY phenomenologies

- Statistics of one year of nominal running should suffice for many phenomenologies

- Knowledge of SM background (and thus of QCD) of paramount importance

- We have yet to find the elusive SUSY But hope prevails

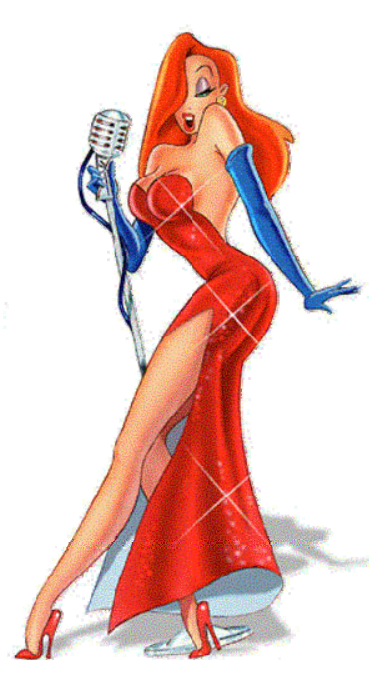




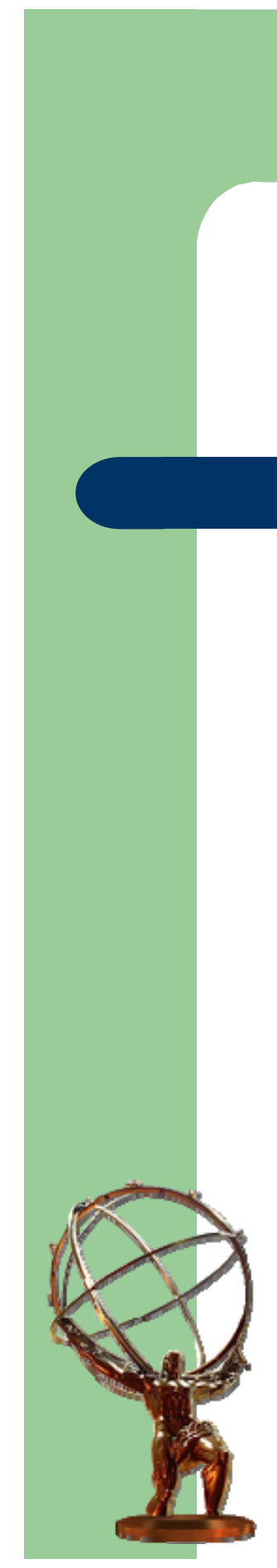

\section{Backup slides}




\section{ATLAS layout}
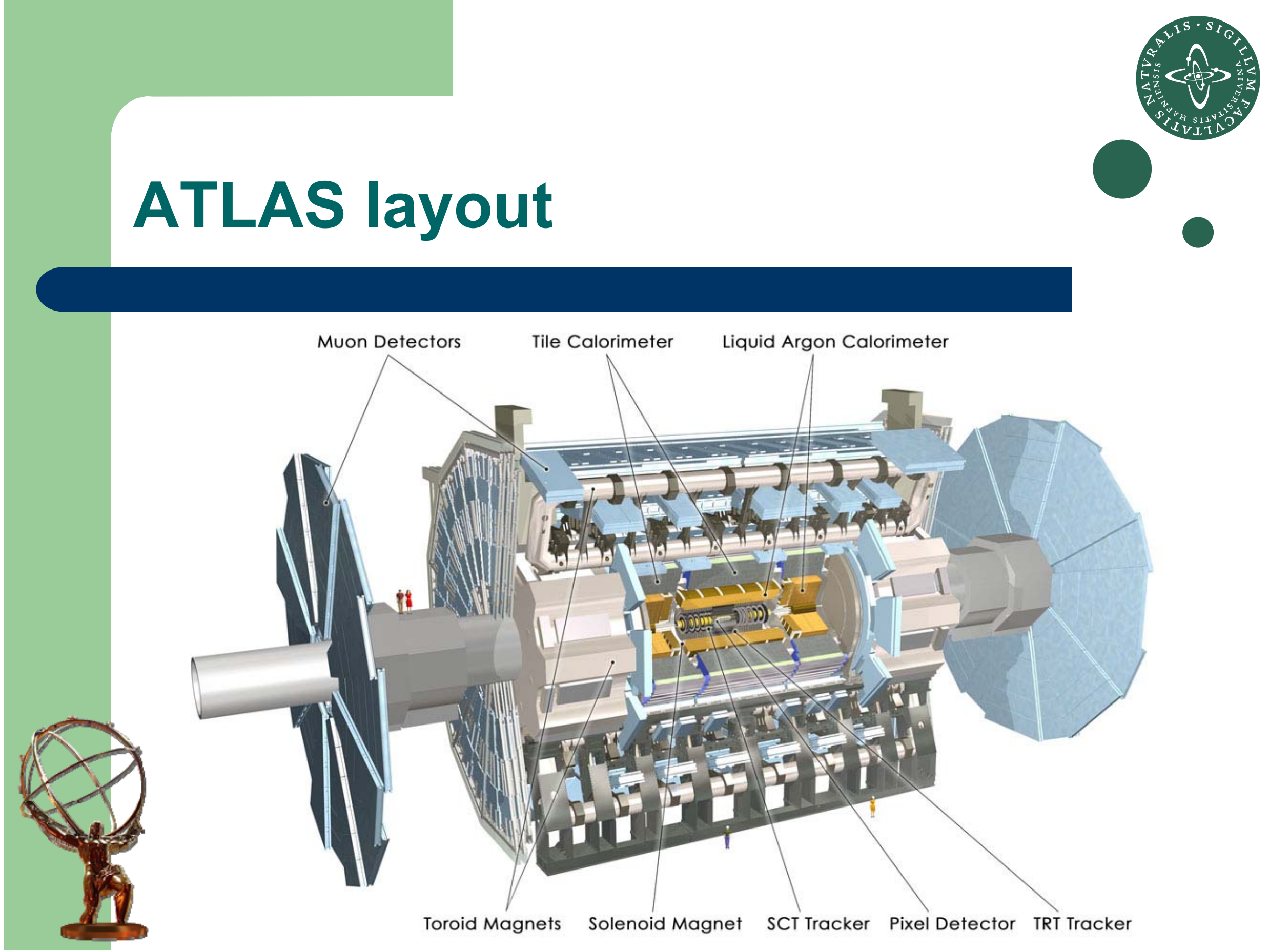


\section{Trigger \& luminosity}

- Low luminosity: $10^{33} \mathrm{~cm}^{-2} \mathrm{~s}^{-1} \rightarrow 10 \mathrm{fb}^{-1} \mathrm{yr}^{-1}$

- High luminosity: $10^{34} \mathrm{~cm}^{-2} \mathrm{~s}^{-1} \rightarrow 100 \mathrm{fb}^{-1} \mathrm{yr}^{-1}$

Trigger rates:

- Raw: 23 collisions / 25 ns (40 mHz)

- LVL1: $75 \mathrm{kHz}$

- HLT: $100 \mathrm{~Hz}$ 
- Sequential decay: $\tilde{q}_{L} \rightarrow \tilde{\chi}_{2}^{0} q \rightarrow \tilde{l}_{R}^{\mp} l_{n e a r}^{ \pm} q \rightarrow \tilde{\chi}_{1}^{0} l_{\text {far }}^{\mp} l_{\text {near }}^{ \pm} q$

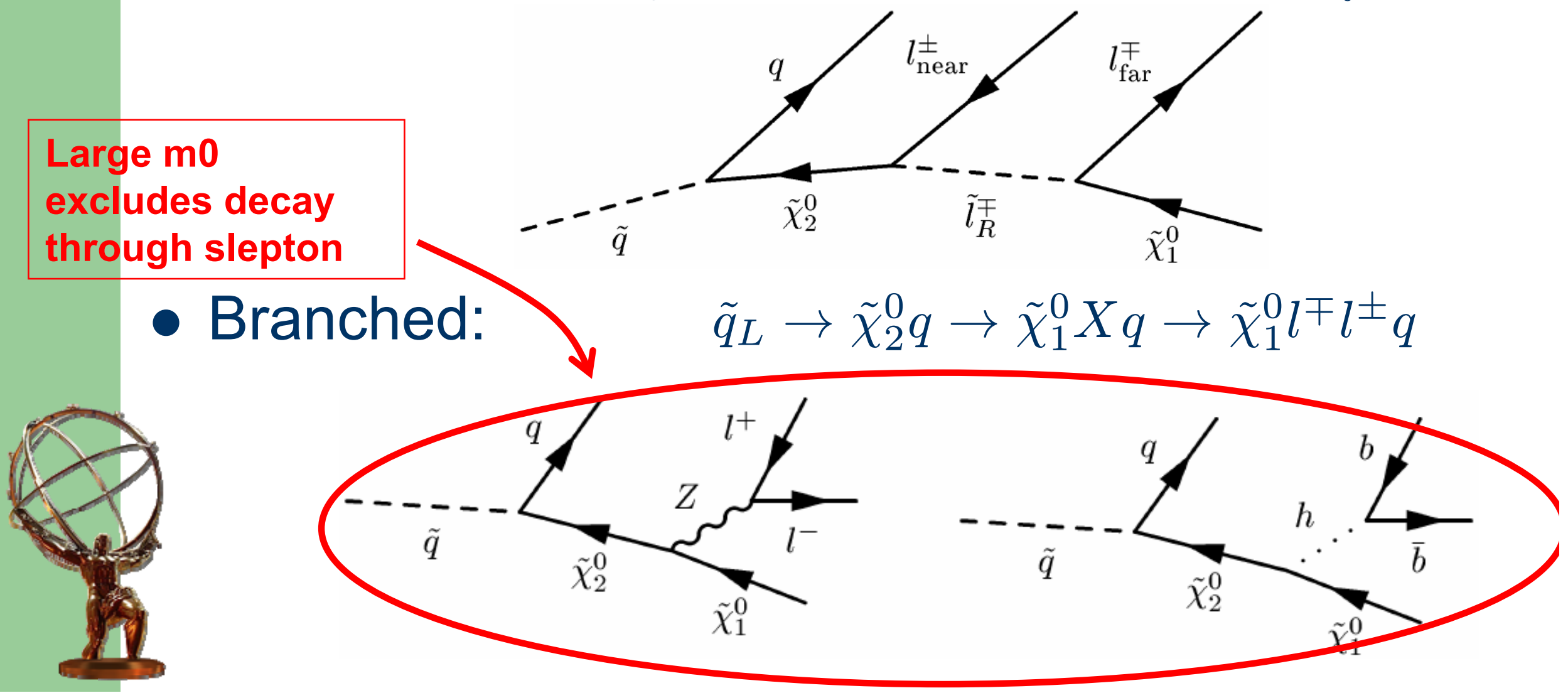


Slide 33

RM18

Figures from J HEP09(2000)004

Rasmus Mackeprang, 8/2/2006 


\section{Edges and thresholds}

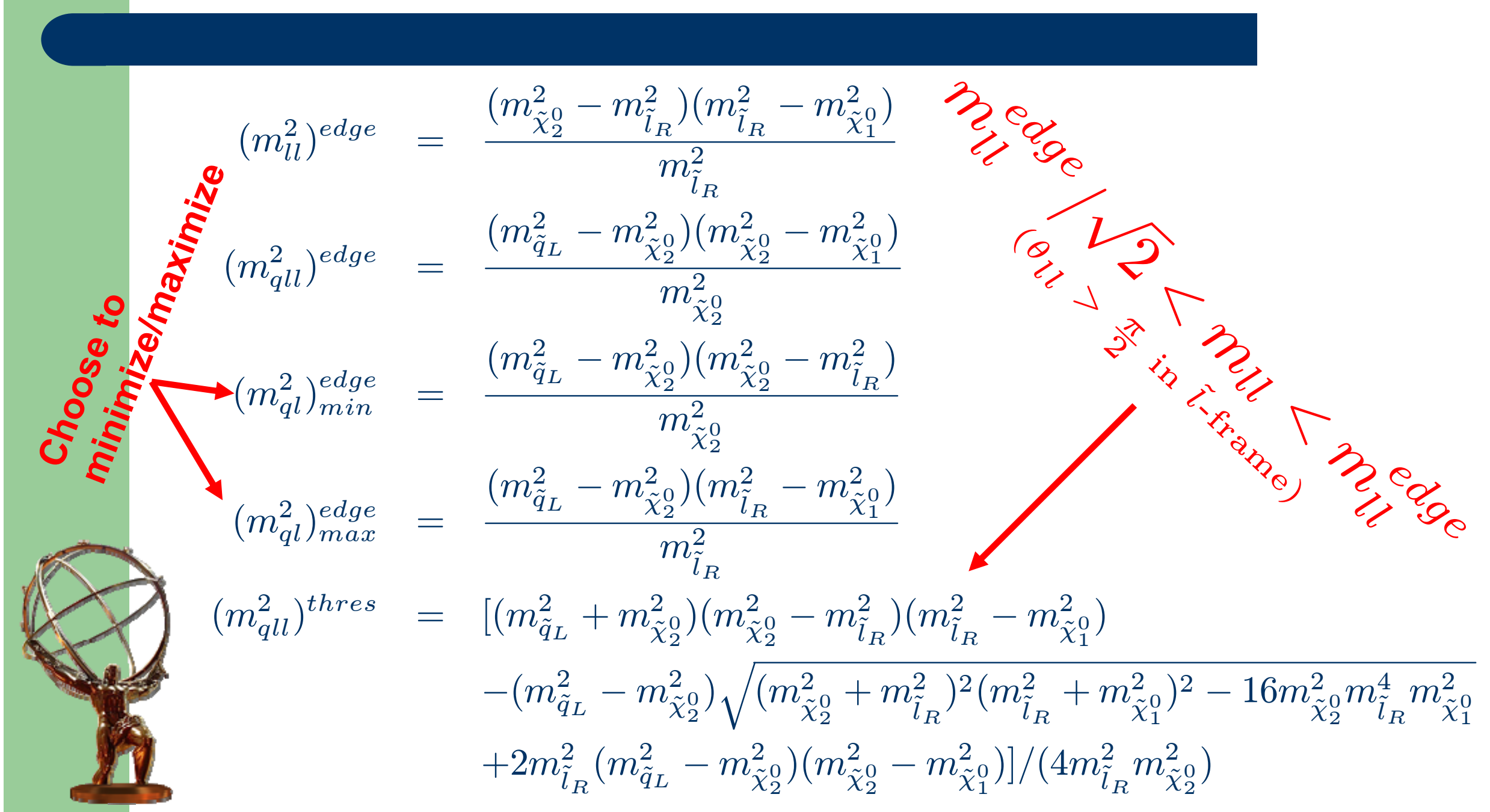


Showing these equations to illustrate the constraints between the SUSY spectrum and the mass edges. Rasmus Mackeprang, 8/4/2006 


\section{qll}

\section{Edge and threshold}
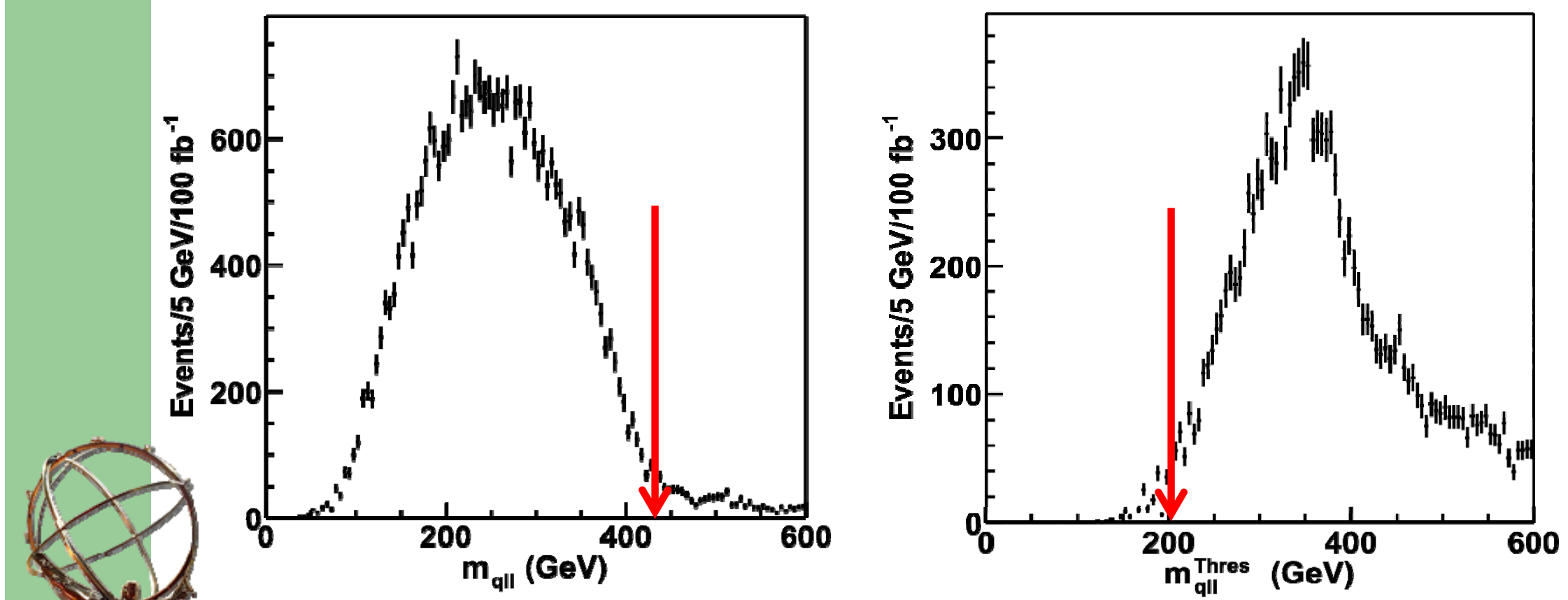

$$
\begin{gathered}
m(q l l)^{\text {edge }}: 431.1^{\text {nominal }} \text { vs. } 431.3^{\text {rec }} \pm 4.3^{\text {syst }} \pm 2.4^{\text {stat }} \\
m(q l l)^{\text {Thres }}: 203.0^{\text {nominal }} \text { vs. } 204.6^{\text {rec }} \pm 2.0^{\text {syst }} \pm 2.8^{\text {stat }}
\end{gathered}
$$


RM16 Just a visual confirmation that the method works Rasmus Mackeprang, 8/4/2006 


\begin{tabular}{lllll}
\hline SP11 & & & \\
SP & & & \\
\hline
\end{tabular}

- Four equations, four masses (in case of universal squark masses) 
Also I should mention that you don't do an inversion at this point, you measure more parameters and do a combined fit as described later. Rasmus Mackeprang, 8/4/2006 


\section{Squark masses @ SPS1A}

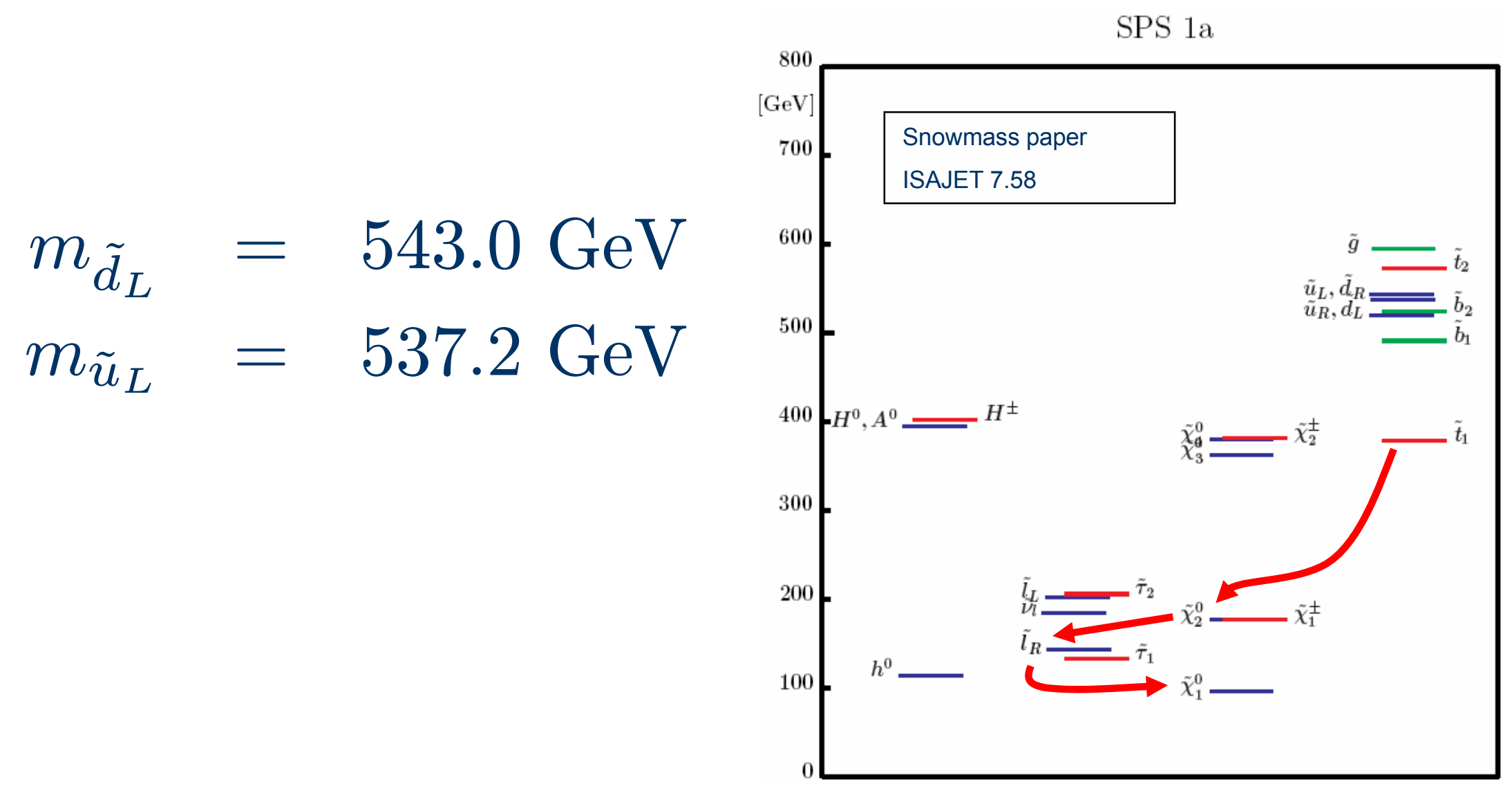




\section{Stop Constraints}

- $m_{\tilde{t}} \in[120 \mathrm{GeV}, 170 \mathrm{GeV}]$

(Strong 1st order phase transition at EW scale)

- $\tilde{t}$ mostly righthanded (LEP)

- $m_{\tilde{t}_{R}}>1 \mathrm{TeV}$ (light Higgs limit)

$\Rightarrow \tilde{t}$ will be produced at the LHC. 


\section{Dark Matter Constraints}

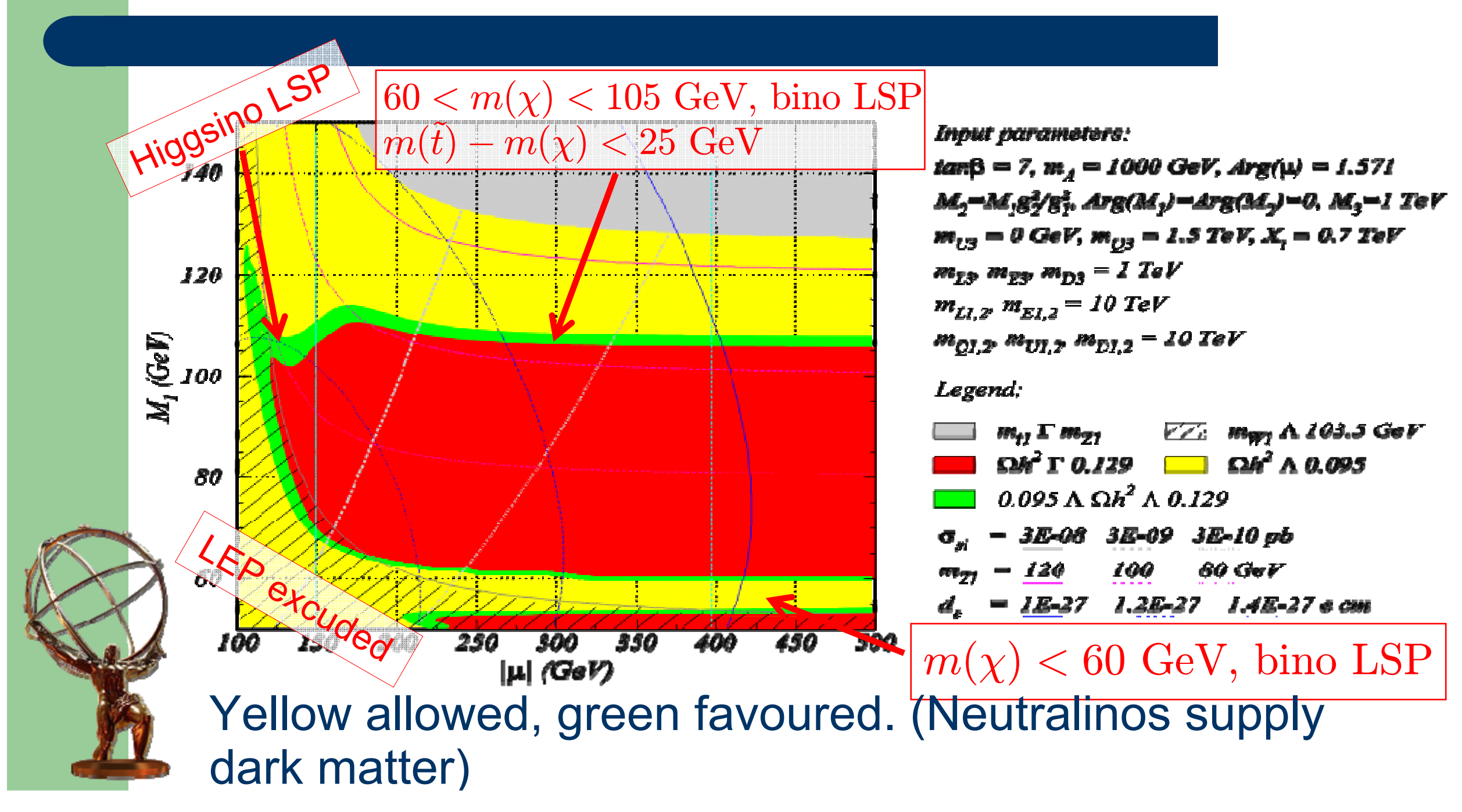


Gotta check w Tomasso if these things mean what I think they mean..

Rasmus Mackeprang, 8/4/2006 


\section{Signal vs Background}

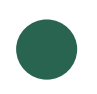

- Difference in shapes in $\mathrm{s} / \mathrm{b}$

- Background shape and normalization crucial.
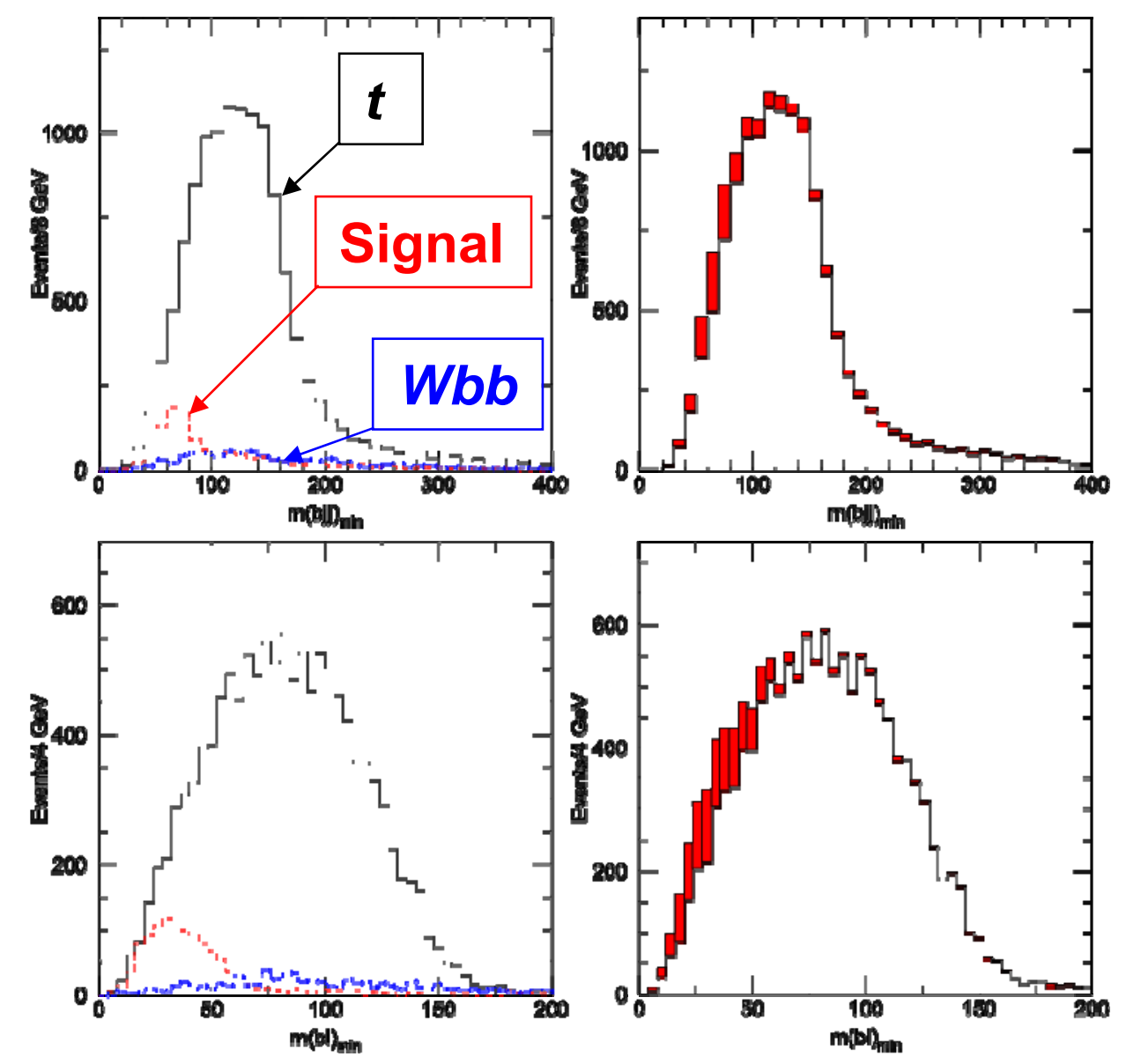


\section{R-hadrons}
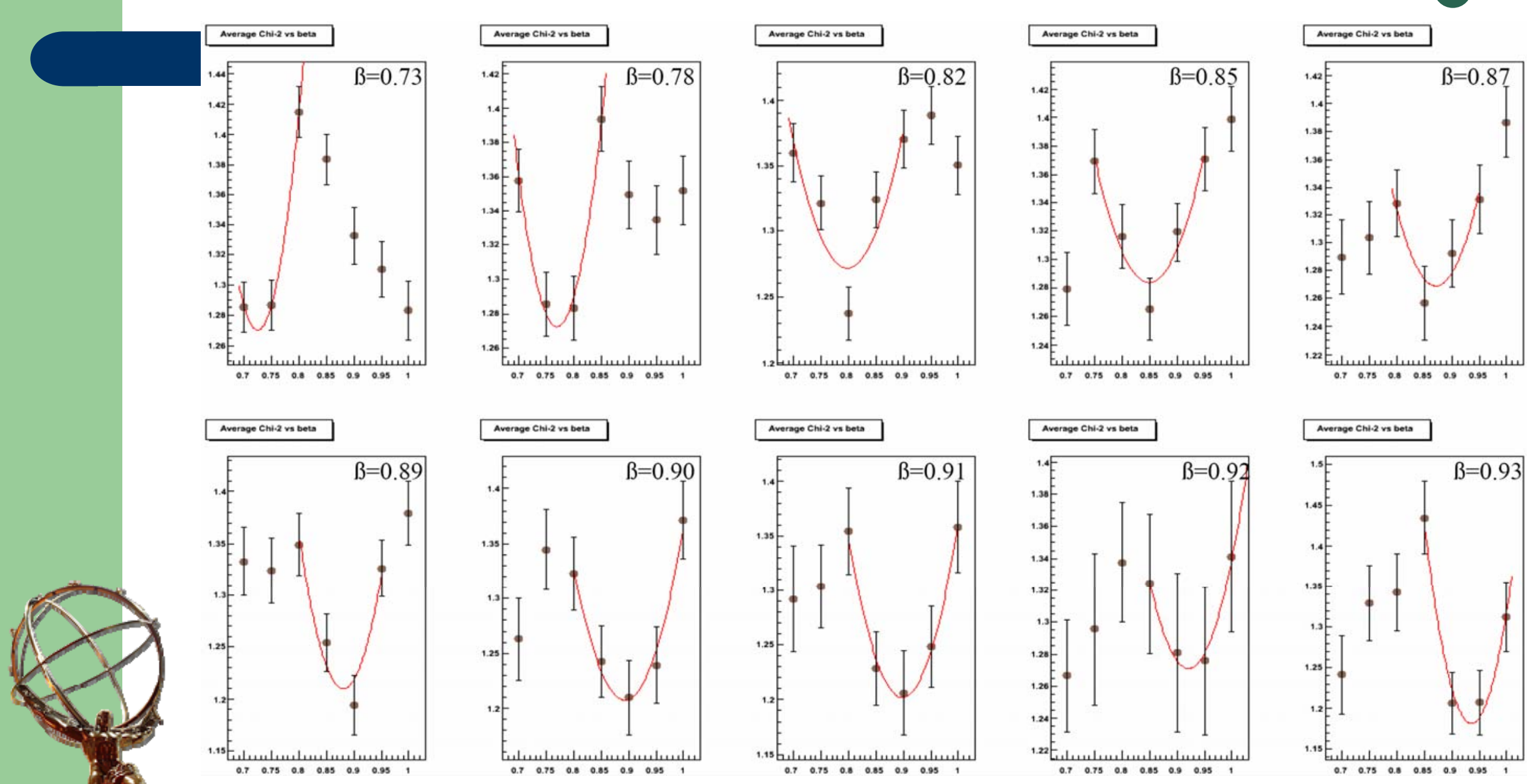

I

Fits a bit unstable $\rightarrow$ Need unbinned method 


\section{R-hadrons}

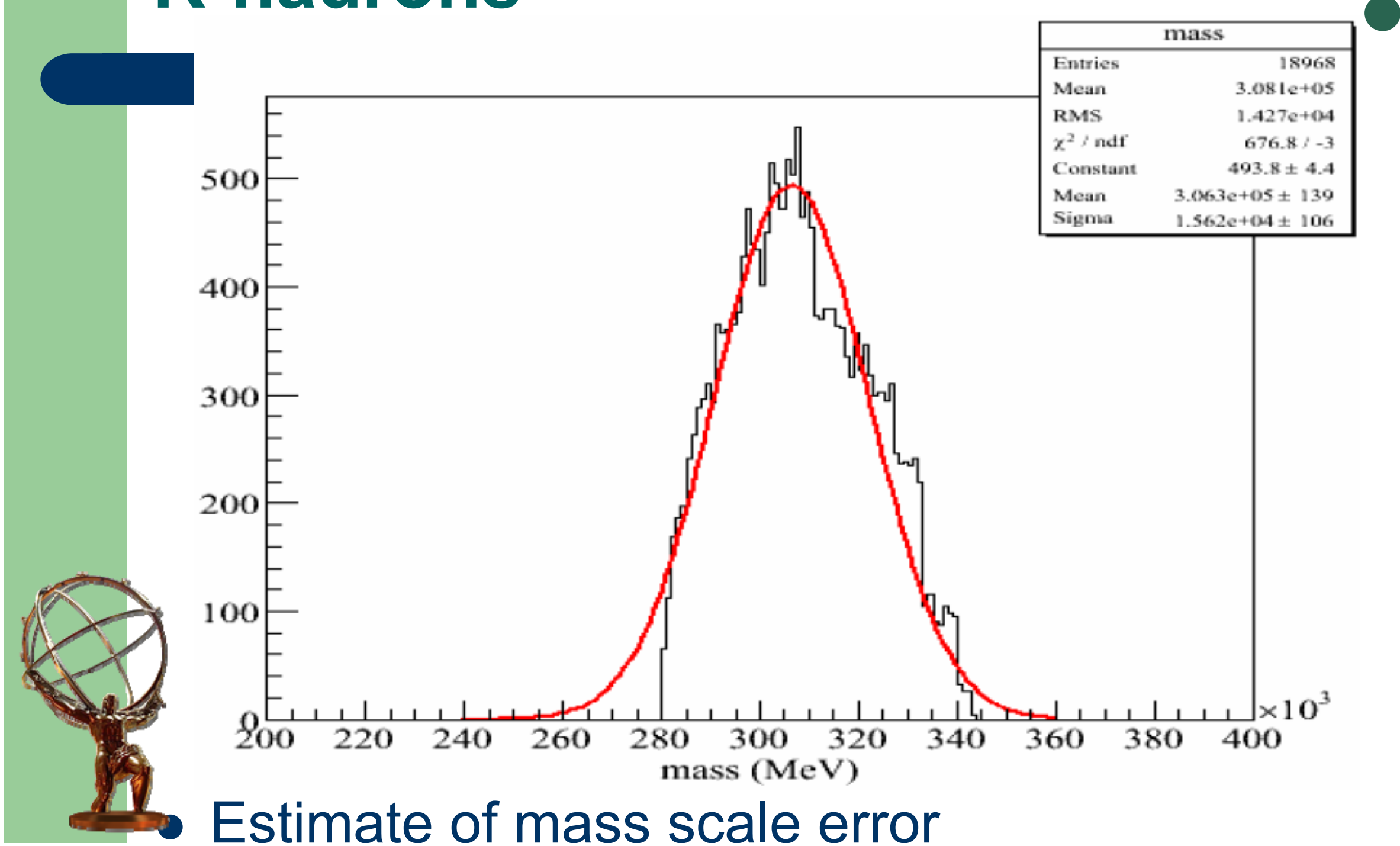




\section{R-meson to baryon conversion}

- $\mathrm{R}^{0}$-mesons with 300 GeV gluino sent through iron

- All mesons end up as baryons.

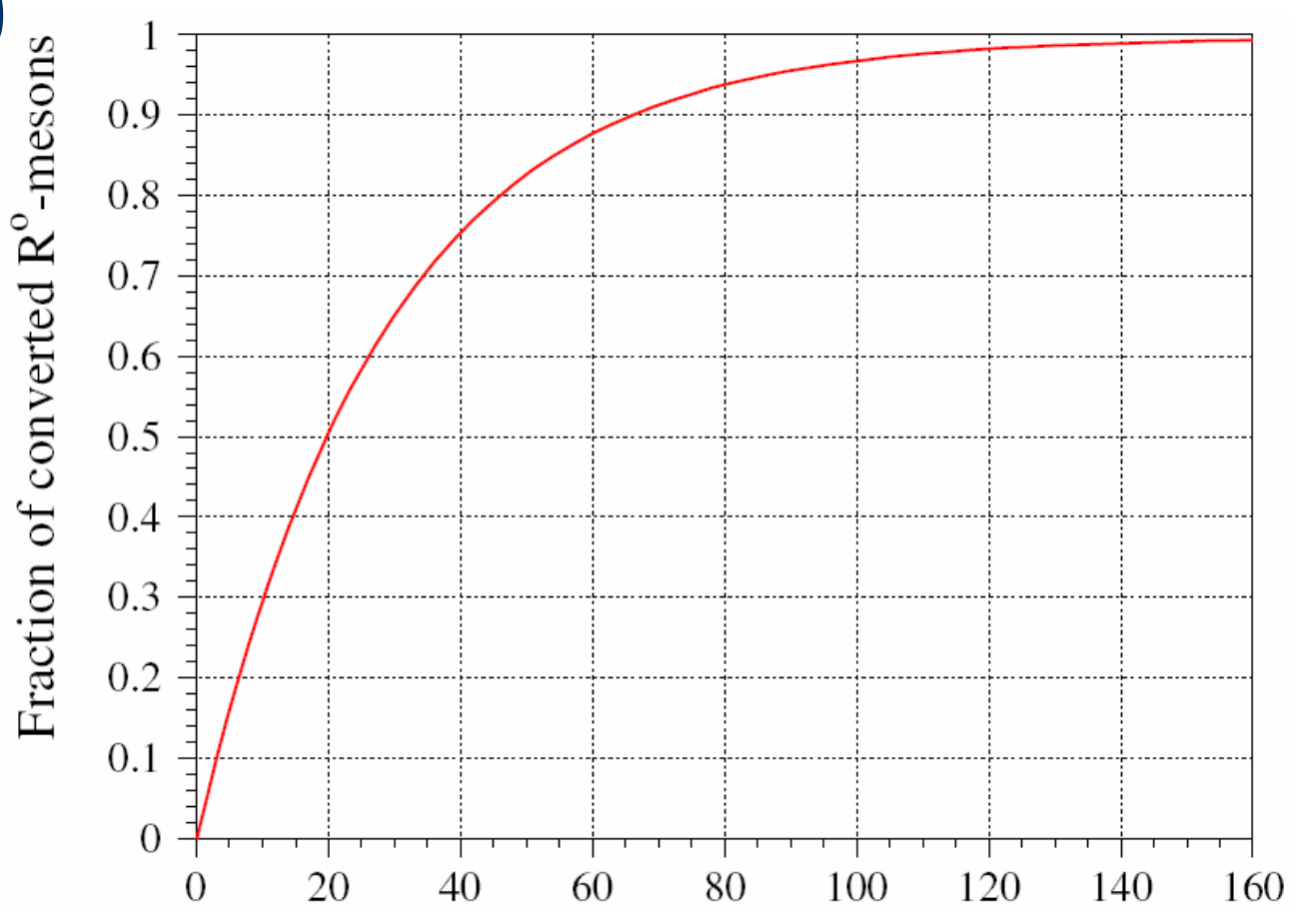




\section{Search Strategies}

- A number of quality cuts can be applied to the tracks used, and R-hadron likeness cuts imposed.

- Sensitivities exceeding the $5 \sigma$ level to metastable gluinos up to $1 \mathrm{TeV}$ in mass has been demonstrated. 
Hvad er lower limit?

Rasmus Mackeprang, $8 / 17 / 2$ 


\section{R-hadrons}

- Assuming flat xsec

(12 mbarn per light quark)

- Assume even weights

- Using phase space function to ensure asymptotic limits for $2 \rightarrow 2$ vs. $2 \rightarrow 3$.

Using parameterised cascade treatment already in Geant4.

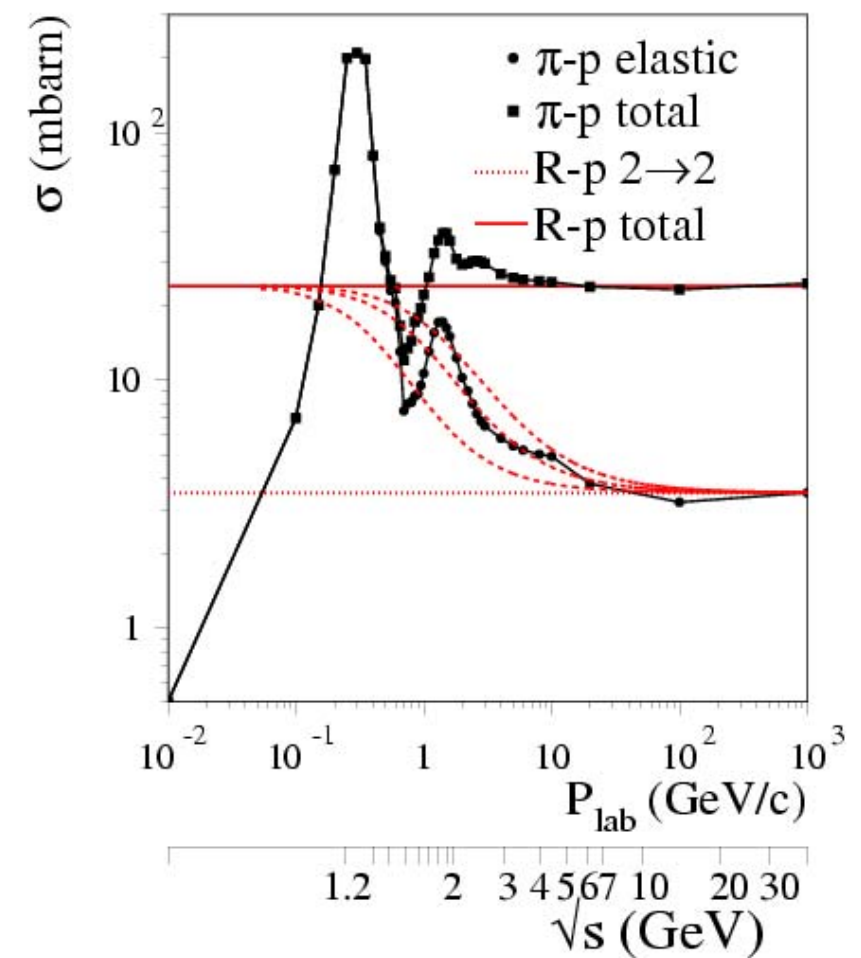

Figure: A.C. Kraan 